\title{
Limites para a Confiabilidade de Sistemas Usados com Componentes com Tempo de Vida $M I F R \mid \Im_{t}$
}

\section{Hélio Arizono}

Tese Apresentada

AO

Instituto de Matemática e Estatística

DA

Universidade de São Paulo

para Obtenção do Grau de Doutor

EM

EstatísticA

Área de Concentração: Probabilidades

Orientador: Prof. Dr. Vanderlei da Costa Bueno

- SÃO PAULO, 1998 - 


\title{
Limites para a Confiabilidade de Sistemas Usados com Componentes com Tempo de Vida $M I F R \mid \Im_{t}$
}

\author{
Este exemplar corresponde à \\ redação final da dissertação \\ devidamente corrigida e defendida \\ por Hélio Arizono e \\ aprovada pela comissão julgadora.
}

São Paulo, 25 de abril de 1998

Banca examinadora

Prof. Dr. Vanderlei da Costa Bueno (Orientador) - IME-USP

Prof. Dr. Jorge Alberto Achcar - ICMSC-USP

Prof. Dr. Mauro Marques - IMEEC-UNICAMP

Prof. Dra. Nancy Lopes Garcia - IMEEC-UNICAMP

Prof. Dr. Enrico Colosimo - UFMG 


\section{RESUMO: LIMITES PARA CONFIABILIDADE DE SISTEMAS USADOS COM COMPONENTES COM TEMPO DE VIDA $M I F R \mid \Im_{t}$}

Consideramos um sistema usado, sua deterioração no tempo e a dependência estatística e estrutural de suas componentes através dos processos de contagem associados com as falhas dos componentes e com a falha do sistema.

Utilizando a teoria dos Martingales para processos pontuais e assumindo que conhecemos um momento ou um percentil obtivemos limites para a confiabilidade de sistemas usados, complexos e com componentes dependentes sob a hipótese de que a distribuição conjunta dos componentes é $M I F R \mid \Im_{t}$ (Multivariate Increasing Failure Rate) relativa a história dos componentes.

\section{ABSTRACT: BOUNDS ON SYSTEM REABILITY OF USED COM- PONENTS UNDER $M I F R \mid \Im_{t}$ ASSUMPTION}

We consider a used system, its time deterioration and the statistical and structural components dependence through the counting processes associated with the components and system failure.

Using martingale theory for counting processes and assuming one moment or one percentile known, we get reliability bounds for used complex system with dependents components under the assumption that the components joint distribution is MIFR (Multivariate Increasing Failure Rate) relative to the components history. 
Agradecimentos

ao Prof. Dr. Vanderlei da Costa Bueno, pela amizade e pela atenciosa orientação, pelas noites e feriados de estudo,

à Vera, ao Samuel e a Cristina, prometo que não haverá mais noites e feriados (pelo menos por alguns meses ...),

aos meus amigos, que ainda não entendem o que eu faço durante as noites e feriados ...,

ao Leonardo, pelo puxão de orelha na hora certa e incentivo,

aos meus pais e minha irmã, pela paciência e compreensão. 


\section{Conteúdo}

Introdução

Capítulo 1. CLASSES DE DISTRIBUIÇÕES DE VIDA CONDICIONADAS À $\sigma$-ÁLGEBRA DO PASSADO OBSERVADO

1.1 Classes de Distribuições $M I F R \mid \Im_{t}$

1.2 Classes de Distribuições $M N B U \mid \Im_{t}$

1.3 Caracterização de Classes de Distribuições Através do

Processo de Risco

Capítulo 2. LIMITES DE CONFIABILIDADE PARA SISTEMAS USADOS

2.1 Formulação Matemática

2.2 Limites para Sistemas com Tempo de Vida $I F R \mid\left(\Im_{t}\right)$

2.3 Limites para Sistemas com Tempo de Vida $N B U \mid\left(\Im_{t}\right)$ 
INTRODUÇÃO 
A Teoria da Confiabilidade considera um sistema $\phi$ com tempo de vida $\tau$, de $n$ componentes $C_{1}, \ldots, C_{n}$, com tempos de vida $S_{1}, \ldots, S_{n}$, respectivamente, dispostos estruturalmente na forma

$$
\tau=\min _{1 \leq j \leq k} \max _{i \in K_{j}} S_{i}
$$

onde $K_{j}, 1 \leq j \leq k$, são os conjuntos de corte minimais, isto é, um conjunto mínimo de componentes cuja falha conjunta causa a falha do sistema.

O cálculo da confiabilidade de sistemas complexos torna-se extremamente complicado e às vezes impossível, pois depende de uma avaliação realista da dependência estatística e estrutural entre os componentes e da sua dinâmica no tempo interpretada por uma noção de idade. A formulação matemática da noção de envelhecimento dá-se através das classes de distribuições com Taxa de Falha Crescente ( $I F R$ ) e Nova é Melhor que Usada $(N B U)$, dentre outras.

O tempo de vida $\tau$ é $I F R$ se e somente se

$$
P(\tau>t+s \mid \tau \geq t) \text { decresce com o tempo } t, t \geq 0 \text {. }
$$

O tempo de vida $\tau$ é $N B U$ se e somente se

$$
P(\tau>t+s) \leq P(\tau>t) P(\tau>s), t \geq 0, s \geq 0 .
$$

Na hipótese de que o tempo de vida do sistema $\tau$ é $I F R(N B U)$, Barlow e Proschan (1981) obteve limites superiores e inferiores para a confiabilidade do sistema assumindo que a média ou algum percentil da distribuição eram conhecidos. Um exemplo destes resultados é dado por:

$$
\bar{F}(t)=\mathbb{P}(\tau>t) \geq \begin{cases}\exp \left\{\frac{-t}{\mu_{1}}\right\}, & \text { se } t<\mu_{1} \\ 0, & \text { se } t \geq \mu_{1}\end{cases}
$$

onde $\tau$ é $I F R$ com média $\mu_{1}$. 
Para o caso em que os tempos de vida dos componentes são $I F R$ o problema permanece sem solução pois, mesmo se todos os componentes do sistema tiverem tempo de vida $I F R$ e forem independentes, o sistema não preservará, necessariamente, a propriedade de tempo de vida $I F R$. O objetivo desta tese é considerar tal questão e produzir limites para sistemas com componentes usados utilizando-se a informação passada

Para abordar tais problemas, ao invés de analisarmos a distribuição conjunta de $\mathbf{S}=\left(S_{1}, \ldots, S_{n}\right)$, analisaremos os processos de contagem associados com as falhas dos componentes,

$$
N_{i}(t)=1_{\left\{S_{i} \leq t\right\}}, i=1, \ldots, n .
$$

e o processo de contagem da falha de sistema $N_{\phi}(t)=1_{\{\tau \leq t\}}$.

Suponha que o comportamento do sistema é monitorado ao nível dos componentes, isto é, a cada tempo $t$ e para todo componente $i$, o pesquisador conhece se o evento $\left\{S_{i} \leq t\right\}$ ocorreu ou não e se $\left\{S_{i} \leq t\right\}$ ocorreu, ele sabe o exato valor de $S_{i}$. A formulação matemática deste conhecimento é a história gerada pelos indicadores dos componentes

$$
\Im_{t}=\sigma\left\{N_{i}(s), \quad s \leq t, \quad i=1, \ldots, n\right\}
$$

Procederemos com o uso da Teoria dos Martingales para Processos Pontuais, que associa a cada processo de contagem $N(t)$ o seu $\Im_{t}$-compensador $A(t)$, um processo previsível, crescente e contínuo à direita $\operatorname{com} A(0)=0$.

No caso clássico, ao considerarmos um objeto com tempo de vida $\tau$, com função de distribuição $F$, a função de risco $R(t)$ acumulada é definida por Barlow e Proschan (1981) como

$$
R(t)=\int_{0}^{t} \frac{d F(s)}{F[s, \infty)}
$$

onde $F[s, \infty)=\mathbb{P}(\tau \geq s)$. 
Pode-se estudar algumas classes de distribuições úteis na Teoria da Confiabilidade através da função de risco. Por exemplo, se $F$ é absolutamente contínua:

- $F$ tem taxa de falha crescente $(I F R)$ se e somente se $R(t)$ é convexa.

- $F$ tem a propriedade $N B U$ se e somente se $R(t+s) \geq R(t)+R(s)$ para todo $t$ e $s \geq 0$.

No conjunto $\{t<\tau\}$ a função de risco coincide com o $\mathcal{G}_{t}$-compensador do processo de contagem $1_{\{\tau \leq t\}}$, onde $\mathcal{G}_{t}=\sigma\left\{1_{\{\tau \leq s\}}, \quad s \leq t\right\}$. Ao tratarmos a dinâmica de vários componentes simultaneamente podemos generalizar suas funções de risco através dos $\Im_{t^{-}}$ compensadores chegando ao Processo de Risco que dependerá fundamentalmente da família de $\sigma$-álgebras $\left(\Im_{t}\right)_{t \geq 0}$.

Consequentemente, para analisarmos a relação entre o processo de risco e as classes de distribuições deveremos considerá-las condicionalmente à família de $\sigma$-álgebras $\left(\Im_{t}\right)_{t \geq 0}$.

Usaremos a classe de distribuições $M I F R \mid \Im_{t}$ (Multivariate Increasing Failure Rate relativa à família de sub- $\sigma$-álgebras $\left(\Im_{t}\right)_{t \geq 0}$ ), definida por Arjas (1981). Com a informação contida em $\left(\Im_{t}\right)_{t \geq 0}$ será possível considerar um sistema usado, sua deterioração no tempo e a dependência estatística e estrutural dos componentes.

Para modelar um sistema usado consideremos um novo espaço de probabilidade $(\Omega, \mathcal{G}, Q) \operatorname{com} \mathcal{G}=\bigvee_{u \geq 0} \mathcal{G}_{u}$, onde

$$
\mathcal{G}_{u}=\sigma\left\{\Im_{t} \bigcup 1_{\{\tau>t+s\}}, \quad 0 \leq s \leq u\right\}
$$

são sub- $\sigma$-álgebras completas e contínuas à direita de $\mathcal{G} \quad=\bigvee_{u \geq 0} \mathcal{G}_{u}$, com $Q\left(A_{1} \times A_{2}\right)=1_{\left\{A_{1}\right\}} \mathbb{P}\left(A_{2} \mid \Im_{t}\right)$ para retângulos $A_{1} \times A_{2} \in \mathcal{G}_{u}$ e $A_{1} \in \Im_{t}$

Podemos interpretar $\mathcal{G}_{u}$ como a história dos componentes até o instante $t$ e o comportamento do sistema no intervalo $(t, t+u]$. Se o processo $N(t)$ conta a falha de um sistema novo, denotaremos $M(u)=\left(N(t+u)-N(t) \mid \Im_{t}\right)$ como o processo que conta a falha de um sistema com idade $t$. 
Um resultado importante (Arjas, 1981) ocorre quando o sistema é formado por componentes com a propriedade $M I F R \mid \Im_{t}$ : o tempo de vida do sistema terá a propriedade $I F R \mid \Im_{t}$.

A partir deste resultado,nesta tese, determinaremos sobre quais condições um sistema formado por componentes $M I F R \mid \Im_{t}$ terá a propriedade $M I F R \mid \mathcal{G}_{u}$ e sob estas condições produziremos limites para a função de sobrevivência do tempo de vida residual $\bar{F}\left(t+u \mid \Im_{t}\right)$.

Assim, sob certas condições podemos generalizaremos os resultados clássicos obtidos por Barlow e Proschan para o caso de sistemas usados, por exemplo:

$$
\bar{F}\left(t+u \mid \Im_{t}\right) \geq \begin{cases}\exp \left\{\frac{-u}{\mathbb{E}\left(\tau-t \mid \Im_{t}\right.}\right\}, & \text { se } u<\mathbb{E}\left(\tau-t \mid \Im_{t}\right) \\ 0, & \text { se } u \geq \mathbb{E}\left(\tau-t \mid \Im_{t}\right)\end{cases}
$$

Nesta tese, também produziremos limites para equações do tipo

$$
\int H(v) d \bar{F}\left(t+v \mid \Im_{t}\right) \text { e } \int \phi(v) \bar{F}\left(t+v \mid \Im_{t}\right) d v
$$

onde $H(v)$ é crescente e convexa e $\phi(v)$ é crescente. 
CAPÍTULO 1

CLASSES DE DISTRIBUIÇÕES DE VIDA CONDICIONADAS À $\sigma$-ÁLGEBRA DO PASSADO OBSERVADO 
1.1 Classes de Distribuições $M I F R \mid \Im_{t}$.

Consideremos um vetor aleatório $\mathbf{S}=\left(S_{1}, \ldots, S_{n}\right), S_{i}>0, i=1, \ldots, n$, representando os tempos de vida dos componentes de um sistema.

No que segue denotaremos por $\theta_{t}\left(S_{i}\right)=\left(S_{i}-t\right)^{+}=\max \left(S_{i}-t, 0\right)$, o tempo de vida residual de $S_{i}$ no instante $t$, e por $\theta_{t} \mathbf{S}=\left(\theta_{t}\left(S_{1}\right), \ldots, \theta_{t}\left(S_{n}\right)\right)$ a representação vetorial.

Começaremos por definir a classe univariada de distribuições de vida $I F R$ relativa a uma família de $\sigma$-álgebras $\left(\Im_{t}\right)_{t \geq 0}$.

Definição 1.1.1: Dizemos que o tempo de vida $\tau$ (ou sua distribuição ) é IFR|( $\Im_{t}$ ) se, para todo $0 \leq t^{*} \leq t$ e $s \in \mathbb{R}$

$$
\mathbb{P}\left\{\theta_{t}(\tau)>s \mid \Im_{t}\right\} \leq \mathbb{P}\left\{\theta_{t^{*}}(\tau)>s \mid \Im_{t^{*}}\right\} \quad \text { q.c. }
$$

ou seja, $\left(\theta_{t}(\tau) \mid \Im_{t}\right)$ é estocasticamente decrescente com o tempo $t$..

A relação entre a definição clássica de $I F R$ e esse novo conceito é dada pela seguinte proposição :

Proposição 1.1.2: Um tempo de vida $\tau$ tem a propriedade $I F R$ se e somente se $\tau$ é $I F R \mid \sigma\left\{1_{\{\tau>s\}}: s \leq t\right\}$.

Prova: Consideremos a função

$$
f(t, s, \omega)=\left\{\begin{array}{cl}
\frac{\bar{F}(t+s)}{\bar{F}(t)}, & \text { se } \bar{F}(t)>0 \text { e } \omega \in\{\tau>t\} \\
0, & \text { caso contrário. }
\end{array}\right.
$$

onde $\bar{F}(t)=1-F(t)$ é a função de sobrevivência do tempo de vida $\tau$. 
Notemos que $\Im_{t}$ é gerado por conjuntos da forma $C=\{\tau>u\}, u \leq t$ e assim,

$$
\begin{aligned}
\int_{C} f(t, s, \omega) \mathbb{P}(d \omega) & =\int_{\{\tau>u\}} \frac{\bar{F}(t+s)}{\bar{F}(t)} 1_{\{\tau>t\}}(\omega) \mathbb{P}(d \omega) \\
& =\frac{\bar{F}(t+s)}{\bar{F}(t)} \int_{\Omega} 1_{\{\tau>t\}}(\omega) \mathbb{P}(d \omega) \\
& =\bar{F}(t+s) \\
& =\mathbb{P}\{\tau>t+s, \tau>u\} \\
& =\mathbb{P}\left\{\theta_{t} \tau>s, C\right\}
\end{aligned}
$$

e utilizando os teoremas de classe monótonas podemos afirmar que $f(t, s, \omega)$ é uma versão de $\mathbb{I P}\left\{\theta_{t} \tau>s \mid \Im_{t}\right\}$ e os dois conceitos são equivalentes.

A extensão multivariada desse conceito dada a seguir utiliza o conceito de conjuntos superiores. Um conjunto $\mathcal{U}$, de $\mathbb{R}^{n}$ é superior se $\mathrm{x} \in \mathcal{U}$ e $\mathrm{y} \geq \mathrm{x}$ então $\mathrm{y} \in \mathcal{U}$.

Definição 1.1.3: Dizemos que $\mathbf{S}=\left(S_{i}\right)_{1 \leq i \leq n}$ tem taxa de falha crescente relativa a $\left(\Im_{t}\right)_{t \geq 0}$ e abreviamos por $M I F R \mid \Im_{t}$ ( Multivariate Increasing Failure Rate relativa a $\sigma$-álgebra $\left.\left(\Im_{t}\right)_{t \geq 0}\right)$ se, para todo conjunto superior aberto $\mathcal{U}$ e todo $0 \leq t^{*} \leq t$, tivermos

$$
\mathbb{P}\left(\theta_{t} \mathrm{~S} \in \mathcal{U} \mid \Im_{t}\right) \leq \mathbb{P}\left(\theta_{t^{*}} \mathrm{~S} \in \mathcal{U} \mid \Im_{t^{*}}\right), \quad \text { q.c. }
$$

Podemos provar que $\mathbf{S}=\left(S_{i}\right)_{1 \leq i \leq n}$ é $M I F R \mid \Im_{t}$ se, e somente se, existe uma versão regular da distribuição condicional $\mathbb{P}^{*}$ e um conjunto de medida nula $N$, tal que em $N^{c}$

$$
\mathbb{P}^{*}\left(\theta_{t} \mathrm{~S} \in \mathcal{U} \mid \Im_{t}\right) \leq \mathbb{P}^{*}\left(\theta_{t^{*}} \mathrm{~S} \in \mathcal{U} \mid \Im_{t^{*}}\right)
$$

para todo $0 \leq t^{*} \leq t$ e todo conjunto superior $\mathcal{U}$. 
Como qualquer esperança condicional é igual (q.c.) a uma integral com respeito a uma versão regular da probabilidade condicional (Breiman, 1968), podemos enunciar a equivalência:

$\mathrm{S}$ é $M I F R \mid \Im_{t}$ se, e somente se, para toda função $f: \mathbb{R}_{+}^{n} \longrightarrow \mathbb{R}$, Borel mensurável, crescente e limitada tivermos

$$
\mathbb{E}\left[f\left(\theta_{t} \mathbf{S} \mid \Im_{t}\right] \leq \mathbb{E}\left[f\left(\theta_{t^{*}} \mathbf{S} \mid \Im_{t^{*}}\right], \quad \text { q.c. },\right.\right.
$$

para todo $0 \leq t^{*} \leq t$

No que segue, discutiremos a preservação da propriedade $M I F R \mid \Im_{t}$ na formação de sistemas monótonos. Provaremos que a propriedade $M I F R \mid \Im_{t}$ dos tempos de vida dos componentes é preservada para o tempo de vida do sistema se a família de $\sigma$-álgebra $\left(\Im_{t}\right)_{t \geq 0}$ for mantida.

Consideremos um sistema $\phi$ com tempo de vida $\tau$, de $n$ componentes $C_{1}, C_{2}, \ldots, C_{n}$, com tempos de vida $S_{1}, S_{2}, \ldots, S_{n}$, respectivamente. O tempo de vida $\tau$ do sistema $\phi$, é completamente determinado pelos tempos de vida dos componentes através da relação funcional

$$
\tau=\phi(\mathbf{S})=\min _{1 \leq j \leq k} \max _{i \in K_{j}} S_{i}
$$

onde $K_{1}, \ldots, K_{k}$ são os conjuntos de cortes minimais da estrutura $\phi$.

Teorema 1.1.4: Nas condições anteriores, se $\mathbf{S}=\left(S_{i}\right)_{1 \leq i \leq n}$ é $M I F R \mid \Im_{t}$ então $\tau$ é $I F R \mid \Im_{t}$.

Prova: Se escrevermos $\tau=\tau(\mathbf{S})$, então 


$$
\begin{aligned}
\theta_{t} \tau(\mathbf{S}) & =(\tau(\mathbf{S})-t)^{+} \\
& =\left(\left(\min _{1 \leq j \leq k} \max _{i \in K_{j}} S_{i}\right)-t\right)^{+} \\
& =\left(\min _{1 \leq j \leq k} \max _{i \in K_{j}}\left(S_{i}-t\right)\right)^{+} \\
& =\min _{1 \leq j \leq k} \max _{i \in K_{j}}\left(S_{i}-t\right)^{+} \\
& =\tau\left(\theta_{t} \mathbf{S}\right) .
\end{aligned}
$$

Como a função $\mathbf{S} \longrightarrow \tau(\mathbf{S})$ é crescente em $\mathbf{S}$ e tomando $f: \mathbb{R} \longrightarrow \mathbb{R}$ é crescente, temos que

$$
f\left(\theta_{t} \tau\right)=f\left(\tau\left(\theta_{t} \mathbf{S}\right)\right)=(f \circ \tau)\left(\theta_{t} \mathbf{S}\right)
$$

onde a função composta $f \circ \tau: \mathbb{R}_{+}^{n} \longrightarrow \mathbb{R}$ é crescente. Assim, como por hipótese $\mathbf{S}$ é $M I F R \mid \Im_{t}$,

$$
\mathbb{E}\left[(f \circ \tau)\left(\theta_{t}(\mathbf{S})\right) \mid \Im_{t}\right] \leq \mathbb{E}\left[(f \circ \tau)\left(\theta_{t^{*}}(\mathbf{S})\right) \mid \Im_{t^{*}}\right], \quad q . c .
$$

para todo $0 \leq t^{*} \leq t$, o que implica que

$$
\mathbb{E}\left[f\left(\theta_{t} \tau\right) \mid \Im_{t}\right] \leq \mathbb{E}\left[f\left(\theta_{t^{*}} \tau\right) \mid \Im_{t^{*}}\right], \quad \text { q.c. },
$$

para todo $0 \leq t^{*} \leq t$ e provamos que $\tau$ é $I F R \mid \Im_{t}$.

O teorema acima parece contradizer um resultado bem conhecido da Teoria Clássica da Confiabilidade: os sistemas monótonos formados por componentes independentes e $I F R$ não tem necessariamente um tempo de vida $I F R$ (Barlow e Proschan (1981)). Consideremos o seguinte exemplo para elucidar a questão:

Exemplo 1.1.5: Sejam $C_{1}$ e $C_{2}$ dois componentes exponenciais independentes de parâmetro 1, em paralelo. Temos que $\tau=S_{1} \vee S_{2}$ é $I F R \mid \sigma\left(1_{\left\{S_{i} \geq s\right\}}, s \leq t, i=1,2\right)$, mas não podemos fazer afirmações a respeito de $\tau$ em relação a $\sigma\left(1_{\{\tau \geq s\}}, s \leq t\right)$; na verdade sabemos que $\tau$ não é $I F R \mid \sigma\left(1_{\{\tau \geq s\}}, s \leq t\right)$, ou seja, $I F R$. 
Cap. 1: Classes de distribuições de vida condicionadas à $\sigma$-álgebra do passado observado

Já demonstramos que a formação de sistemas monótonos preserva a propriedade $M I F R \mid \Im_{t}$ de seus componentes e mostramos que o mesmo não acontece se mudamos a família de $\sigma$-álgebras. Seria desejável encontrar condições sob as quais sistemas monótonos preservassem a propriedade $M I F R \mid \Im_{t}$ de seus componentes, mas relativamente a uma família de $\sigma$-álgebras

$\left(\mathcal{G}_{t}\right)_{t \geq 0}$ contida na família $\left(\Im_{t}\right)_{t \geq 0}$. Como exemplo, consideremos

$\Im_{t}=\sigma\left\{1_{\left\{S_{i}>s\right\}}, s \leq t, 1 \leq i \leq n\right\}$, a $\sigma$-álgebra gerada pelos indicadores dos componentes e

$\mathcal{G}_{t}=\sigma\left\{1_{\{\tau>s\}}, s \leq t\right\}$, a $\sigma$-álgebra gerada pelo sistema.

Notemos que $\{\tau>s\}=\bigcap_{k} \bigcup_{i \in K_{j}}\left\{S_{i}>s\right\}$, de maneira que $\mathcal{G}_{t} \subset \Im_{t}, t \geq 0$.

As condições desejáveis estão no seguinte teorema:

Teorema 1.1.6: Suponhamos que S seja $M I F R \mid \Im_{t}$ e que $\mathcal{G}_{t} \subset \Im_{t}$ seja tal que, para todo $t \geq 0, \theta_{t} \tau$ seja independente de $\Im_{t}$, dado $\mathcal{G}_{t}$. Então $\tau$ é $I F R \mid \mathcal{G}_{t}$.

Prova: Pelo Teorema 1.1.4, $\tau$ é $I F R \mid \Im_{t}$ de maneira que para todo $s \in \mathbb{R}$ e $0 \leq t^{*} \leq t$

$$
\mathbb{P}\left(\theta_{t} \tau>s \mid \Im_{t}\right) \leq \mathbb{P}\left(\theta_{t^{*}} \tau>s \mid \Im_{t^{*}}\right), \quad \text { q.c. }
$$


Como $\tau$ é independente de $\Im_{t}$, dado $\mathcal{G}_{t}$, podemos afirmar que

$$
\begin{aligned}
\mathbb{P}\left(\theta_{t} \tau>s \mid \mathcal{G}_{t}\right) & =\mathbb{P}\left(\theta_{t} \tau>s \mid \Im_{t}\right) \\
& \leq \mathbb{P}\left(\theta_{t^{*}} \tau>s \mid \Im_{t^{*}}\right) \\
& =\mathbb{P}\left(\theta_{t^{*}} \tau>s \mid \mathcal{G}_{t^{*}}\right), \quad \text { q.c. }
\end{aligned}
$$

Um exemplo não trivial em que as condições do teorema estão satisfeitas ocorre no caso em que o sistema é formado por componentes em série.

Estes conceitos preservam a maioria das propriedades que poderiam ser chamadas de desejáveis para qualquer extensão dos conceitos clássicos da Teoria da Confiabilidade. Pode-se provar que a classe $I F R \mid \Im_{t}$ é equivalente à classe de distribuições de vida $I F R$ quando $\Im_{t}$ é a $\sigma$-álgebra gerada pelo indicador do tempo de vida. Mostraremos agora que qualquer subvetor de um vetor $M I F R \mid \Im_{t}$ também é $M I F R \mid \Im_{t}$ e que a composição de dois vetores $M I F R \mid \Im_{t}$, independentes, é $M I F R \mid \Im_{t}$.

Teorema 1.1.7: Suponhamos que $\mathbf{S}=\left(S_{i}\right)_{1 \leq i \leq n}$ é $M I F R \mid \Im_{t}$. Então, qualquer subvetor $\mathbf{S}_{0}=\left(S_{i}\right)_{i \in I_{0}}$ com $I_{0} \subset\{1, \ldots, n\}$ é $M I F R \mid \Im_{t}$. Em particular, cada $S_{i}$ é $I F R \mid \Im_{t}$.

Prova: Segue facilmente pois qualquer conjunto superior $\mathcal{U}_{0}$ em $\mathbb{R}^{\operatorname{card}\left(I_{0}\right)}$ pode ser escrito como $\mathcal{U}=\left\{\mathbf{x} \in \mathbb{R}^{n}:\left(x_{i}\right)_{i \in I_{0}} \in \mathcal{U}_{0}\right\}$, um conjunto superior em $\mathbb{R}^{n}$ e portanto, se $0 \leq t^{*} \leq t$, temos que

$$
\begin{gathered}
\mathbb{P}\left(\theta_{t} \mathrm{~S}_{0} \in \mathcal{U}_{0} \mid \Im_{t}\right)=\mathbb{I P}\left(\theta_{t} \mathbf{S} \in \mathcal{U} \mid \Im_{t}\right) \\
\leq \mathbb{P}\left(\theta_{t^{*}} \mathbf{S} \in \mathcal{U} \mid \Im_{t^{*}}\right)=\mathbb{P}\left(\theta_{t^{*}} \mathrm{~S}_{0} \in \mathcal{U}_{0} \mid \Im_{t^{*}}\right), \quad q . c . .
\end{gathered}
$$


No teorema anterior, a família de sub- $\sigma$ 'algebras $\left(\Im_{t}\right)_{t \geq 0}$ é a mesma para $\mathbf{S}$ e $\mathbf{S}_{\mathbf{0}}$. Seria interessante verificar se $\left(\Im_{t}\right)_{t \geq 0}$ pode ser alterada para uma família de sub $\sigma$-álgebras $\left(\mathcal{G}_{t}\right)_{t \geq 0}$ com $\mathcal{G}_{t} \subset \Im_{t}$ para todo $t \geq 0$, com o propósito de analisarmos $\left(\theta_{t} \mathbf{S}_{0} \mid \mathcal{G}_{t}\right)$.

Note que, em geral, se $\mathcal{G}_{t} \subset \Im_{t}$, a implicação $\mathbf{S}$ é $M I F R \mid \Im_{t} \Longrightarrow \mathbf{S}$ é $M I F R \mid \mathcal{G}_{t}$ não é verdadeira; basta lembrarmos que sistemas monótonos de componentes $M I F R \mid \Im_{t}$ é $M I F R \mid \Im_{t}$ mas não é $M I F R \mid \mathcal{G}_{t}$, onde

$$
\begin{gathered}
\Im_{t}=\sigma\left\{1_{\left\{S_{i}>s\right\}}, s \leq t, i=1, \ldots, n\right\} \quad e \\
\mathcal{G}_{t}=\sigma\left\{1_{\{\tau>s\}}, s \leq t\right\} . \\
\frac{\sqrt{Y_{T+c}}}{y} V_{Y_{i}+t}
\end{gathered}
$$

A proposição a seguir descreve um caso em que vale esta afirmação . Basicamente assumimos que, devido a independência, uma parte da $\sigma$-álgebra é redundante para o subvetor.

Teorema 1.1.8: Suponhamos que $\mathbf{S}=\left(S_{i}\right)_{1 \leq i \leq n}$ seja $M I F R \mid \Im_{t}$ onde $\Im_{t}$ é a $\sigma$-álgebra gerada pelos indicadores dos componentes. Seja $I_{0} \subset\{1, \ldots, n\}$ e $\mathcal{G}_{t}$ a $\sigma$-álgebra gerada pelos indicadores dos componentes que pertencem a $I_{0}$. Se $\mathrm{S}_{0}=\left(S_{i}\right)_{i \in I_{0}}$ e $\mathrm{S}_{1}=$ $\left(S_{i}\right)_{i \in I_{0}^{c}}$ são independentes, então $\mathrm{S}_{0}$ é $M I F R \mid \mathcal{G}_{t}$.

Prova: Seja $\aleph_{t}=\sigma\left\{1_{\left\{S_{i}>s\right\}}, s \leq t, i \in I_{0}^{c}\right\}$, assim $\Im_{t}=\mathcal{G}_{t} \vee \aleph_{t}$. Denotemos a independência de $\Gamma$ e $\Delta$ por $\Gamma \coprod \Delta$ e a independência condicional, dado $\Lambda$, por $\Gamma \coprod \Delta \mid \Lambda$.

Por hipótese $\mathcal{G}_{\infty} \coprod \aleph_{\infty}$ e portanto $\mathcal{G}_{\infty} \coprod \aleph_{t}$. Pelo fato de que $\mathcal{G}_{t} \subset \mathcal{G}_{\infty}$ temos então que $\mathcal{G}_{\infty}\left\lfloor\aleph_{t} \mid \mathcal{G}_{t}\right.$ (pois para qualquer $B \in \aleph_{t}, \mathbb{P}\left(B \mid \mathcal{G}_{t} \vee \mathcal{G}_{\infty}\right)=\mathbb{P}\left(B \mid \mathcal{G}_{\infty}\right)=\mathbb{P}(B)=$ $\mathbb{P}\left(B \mid \mathcal{G}_{t}\right) \quad$ q.c. $)$. Portanto, para qualquer $A \in \mathcal{G}_{\infty}$, temos $\mathbb{P}\left(A \mid \mathcal{G}_{t} \vee \aleph_{t}\right)=\mathbb{P}\left(A \mid \mathcal{G}_{t}\right) \quad$ q.c. e em particular, temos que

$$
\mathbb{P}\left(\theta_{t} \mathrm{~S}_{0} \in \mathcal{U} \mid \Im_{t}\right)=\mathbb{P}\left(\theta_{t} \mathrm{~S}_{0} \in \mathcal{U} \mid \mathcal{G}_{t}\right) \quad \text { q.c.. }
$$


Cap. 1: Classes de distribuições de vida condicionadas à $\sigma$-álgebra do passado observado

Similarmente,

$$
\mathbb{P}\left(\theta_{t^{*}} \mathbf{S}_{0} \in \mathcal{U} \mid \Im_{t^{*}}\right)=\mathbb{P}\left(\theta_{t^{*}} \mathbf{S}_{0} \in \mathcal{U} \mid \mathcal{G}_{t^{*}}\right), \quad \text { q.c. }
$$

e portanto pelo Teorema 1.1 .7

$$
\mathbb{I P}\left(\theta_{t} \mathbf{S}_{0} \in \mathcal{U} \mid \mathcal{G}_{t}\right) \leq \mathbb{P}\left(\theta_{t^{*}} \mathbf{S}_{0} \in \mathcal{U} \mid \mathcal{G}_{t^{*}}\right), \quad \text { q.c. }
$$

se $0 \leq t^{*} \leq t$, onde $\mathcal{U}$ é um conjunto superior aberto.

O próximo teorema mostra que se dois vetores independentes são, separadamente, $M I F R \mid \Im_{t}$ então a junção dos dois vetores também é $M I F R \mid \Im_{t}$.

Teorema 1.1.9: Suponhamos que $\mathbf{S}_{1}$ e $\mathbf{S}_{2}$ sejam $M I F R \mid \Im_{t}$ e que para cada $t$, $\theta_{t} \mathbf{S}_{1}$ e $\theta_{t} \mathbf{S}_{2}$ sejam independentes, dado $\Im_{t}$. Então $\mathbf{S}=\left(\mathbf{S}_{1}, \mathbf{S}_{2}\right)$ também é $M I F R \mid \Im_{t}$.

Prova: Para $\omega \notin N$ ( $N$ conjunto de medida nula), escrevamos a versão regular da probabilidade condicional

$$
\mathbb{I P}\left(\theta_{u} \mathbf{S}_{\mathbf{i}} \in . \mid \Im_{u}\right)(\omega)=\mathbf{H}_{\mathbf{i}}(u, . ; \omega), \quad i=1,2
$$

Seja $d_{i}=\operatorname{card}\left(I_{i}\right), i=1,2$ e seja $f: \mathbb{R}^{d_{1}+d_{2}} \longrightarrow \mathbb{R}$ uma função crescente. Assim, para $0 \leq t^{*} \leq t$

$$
\begin{aligned}
& \mathbb{E}\left[f\left(\theta_{t}\left(\mathbf{S}_{1}, \mathbf{S}_{2}\right)\right) \mid \Im_{t}\right]=\int\left[\int f\left(\mathbf{x}_{1}, \mathbf{x}_{2}\right) \mathbf{H}_{1}\left(t, d \mathbf{x}_{1} ; .\right)\right] \mathbf{H}_{2}\left(t, d \mathbf{x}_{2} ; .\right) \\
& \leq \int\left[\int f\left(\mathbf{x}_{1}, \mathbf{x}_{2}\right) \mathbf{H}_{1}\left(t^{*}, d \mathbf{x}_{1} ; .\right)\right] \mathbf{H}_{2}\left(t, d \mathbf{x}_{2} ; .\right) \\
& \leq \int\left[\int f\left(\mathbf{x}_{1}, \mathbf{x}_{2}\right) \mathbf{H}_{1}\left(t^{*}, d \mathbf{x}_{1} ; .\right)\right] \mathbf{H}_{2}\left(t^{*}, d \mathbf{x}_{2} ; .\right) \\
&=\mathbb{E}\left[f\left(\theta_{t^{*}}\left(\mathbf{S}_{1}, \mathbf{S}_{2}\right)\right) \mid \Im_{t^{*}}\right] \\
&-14-
\end{aligned}
$$


A primeira desigualdade vem do fato de que $f\left(\mathbf{x}_{1}, \mathbf{x}_{2}\right)$ é crescente em $\mathbf{x}_{1}$ para $\mathbf{x}_{\mathbf{2}}$ fixado, $\int f\left(\mathbf{x}_{1}, \mathbf{x}_{2}\right) \mathbf{H}_{1}\left(u, d \mathbf{x}_{1} ;.\right)=\mathbb{E}\left[f\left(\theta_{u}\left(\mathbf{S}_{1}, \mathbf{x}_{2}\right)\right) \mid \Im_{u}\right] \quad q . c .$, e $\mathbf{S}_{1}$ é $M I F R \mid \Im_{t}$.

A segunda desigualdade ocorre pois $\int f\left(\mathbf{x}_{1}, \mathbf{x}_{2}\right) \mathbf{H}_{1}\left(t^{*}, d \mathbf{x}_{1} ;\right)$ é crescente em $\mathbf{x}_{2}$ e $\mathrm{S}_{2}$ é $M I F R \mid \Im_{t}$.

O teorema anterior exige uma única família de $\sigma$-álgebras para $\mathbf{S}, \mathbf{S}_{\mathbf{1}}$ e $\mathbf{S}_{\mathbf{2}}$. Entretanto, uma situação mais interessante ocorre quando consideramos $\mathbf{S}_{1}$ e $\mathbf{S}_{2}$ em relação às suas próprias $\sigma$-álgebras que combinadas geram a $\sigma$-álgebra de $\mathbf{S}=\left(\mathbf{S}_{1}, \mathbf{S}_{2}\right)$.

Teorema 1.1.10: Suponhamos que $\mathbf{S}_{1}=\left(S_{i}\right)_{i \in I_{1}}$ e $\mathbf{S}_{\mathbf{2}}=\left(S_{i}\right)_{i \in I_{2}}$ sejam independentes. Se $\mathbf{S}_{\mathbf{j}}$ é $M I F R \mid \Im_{t}^{j}$ onde $\Im_{t}^{j} \subset \mathcal{G}_{t}^{j}=\sigma\left\{1_{\left\{S_{i}>s\right\}}, s \leq t, i \in I_{j}\right\}, j=1,2$ então $\mathbf{S}=\left(\mathbf{S}_{1}, \mathbf{S}_{2}\right)$ é $M I F R \mid\left(\Im_{t}^{1} \vee \Im_{t}^{2}\right)$.

Prova: Da proposição anterior, observamos que se $\mathcal{G}_{\infty}^{1} \amalg \mathcal{G}_{\infty}^{2}$ então $\mathcal{G}_{\infty}^{1} \amalg \Im_{t}^{2}$ e $\mathcal{G}_{\infty}^{1} \amalg \Im_{t}^{2} \mid \Im_{t}^{1}$ e portanto

$$
\mathbb{I P}\left(\theta_{t} \mathbf{S}_{1} \in \mathcal{U} \mid \Im_{t}^{1} \vee \Im_{t}^{2}\right)=\mathbb{P}\left(\theta_{t} \mathbf{S}_{1} \in \mathcal{U} \mid \Im_{t}^{1}\right) \quad \text { q.c. }
$$

e, de maneira análoga,

$$
\mathbb{P}\left(\theta_{t} \mathbf{S}_{2} \in \mathcal{U} \mid \Im_{t}^{1} \vee \Im_{t}^{2}\right)=\mathbb{I P}\left(\theta_{t} \mathbf{S}_{2} \in \mathcal{U} \mid \Im_{t}^{2}\right) \quad \text { q.c. }
$$

Portanto $\mathbf{S}_{1}$ e $\mathbf{S}_{2}$ são $M I F R \mid\left(\Im_{t}^{1} \vee \Im_{t}^{2}\right)$ e como $G_{\infty}^{1} \amalg G_{\infty}^{2}$ implica $G_{\infty}^{1} \amalg G_{\infty}^{2} \mid \Im_{t}^{1} \vee$ $\Im_{t}^{2}$, podemos aplicar o teorema anterior e obter o resultado desejado.

Corolário 1.1.11: Suponhamos que $S_{1}, \ldots, S_{n}$ sejam independentes e $I F R$. Então $\mathbf{S}=\left(S_{i}\right)_{1 \leq i \leq n}$ é $M I F R \mid \sigma\left\{1_{\left\{S_{i}>s\right\}}, s \leq t, 1 \leq i \leq n\right\}$, ou seja, um conjunto de tempos $I F R$ e independentes é $M I F R$ com relação a $\sigma$-álgebra gerada pelos componentes. 
1.2 Classes de distribuições $M N B U \mid \Im_{t}$.

O tempo de vida de um objeto $\tau$ é $N B U$ (New Better than Used) se a variável aleatória $(\tau-t \mid\{\tau>t\})$, condicionada em $\{\tau>t\}$, é estocasticamente menor do que $\tau$. A extensão dessa definição segue das mesmas idéias utilizadas na definição de $M I F R \mid \Im_{t}$.

Definição 1.2.1: Dizemos que $\mathbf{S}=\left(S_{i}\right)_{1 \leq i \leq n}$ é Multivariate New Better than Used relativa à família $\left(\Im_{t}\right)_{t \geq 0}$ e abreviamos por $M N B U \mid \Im_{t}$ se, para todo conjunto superior aberto $\mathcal{U}$ e para todo $t \geq 0$

$$
\mathbb{P}\left(\theta_{t} \mathrm{~S} \in \mathcal{U} \mid \Im_{t}\right) \leq \mathbb{P}\left(\mathbf{S} \in \mathcal{U} \mid \Im_{0}\right), \quad \text { q.c. }
$$

No caso univariado, dizemos que $\tau$ é $N B U \mid \Im_{t}$ se, para $0 \leq t^{*} \leq t$ e $s \in \mathbb{R}$,

$$
\mathbb{P}\left(\theta_{t}(\tau)>s \mid \Im_{t}\right) \leq \mathbb{P}\left(\tau>s \mid \Im_{0}\right), \quad \text { q.c. }
$$

Em particular, se $\Im_{t}=\sigma\left\{1_{\{\tau>s\}}: s \leq t\right\}$, a definição $N B U \mid \Im_{t}$ é equivalente à definição clássica $N B U$.

É fácil provar que a classe $M I F R \mid \Im_{t}$ está contida na classe $M N B U \mid \Im_{t}$; basta tomar $t^{*}=0$ na definição 1.1.3.

Notamos também que todos os resultados provados para a classe de distribuições $M I F R \mid \Im_{t}$ valem para a classe $M N B U \mid \Im_{t}$. Contudo, a classe de distribuições $M N B U \mid \Im_{t}$ é preservada quando restringimos a definição a uma família de $\sigma$-álgebras $\left(\mathcal{G}_{t}\right)_{t \geq 0}$ contida em $\left(\Im_{t}\right)_{t \geq 0}$. (isto não acontece na classe $M I F R \mid \Im_{t}$ ). 
Teorema 1.2.2: Suponhamos que S é $M N B U \mid \Im_{t}$. Se $\left(\mathcal{G}_{t}\right)_{t \geq 0}$ é uma família crescente de $\sigma$-álgebras com $\mathcal{G}_{t} \subset \Im_{t}$, para todo $t>0$ e $\mathcal{G}_{0}=\Im_{0}$, então S é $M N B U \mid \mathcal{G}_{t}$.

\section{Prova:}

$$
\begin{aligned}
\mathbb{P}\left(\theta_{t} \mathrm{~S} \in \mathcal{U} \mid \mathcal{G}_{t}\right) & =\mathbb{E}\left[\mathbb{P}\left(\theta_{t} \mathrm{~S} \in \mathcal{U} \mid \Im_{t}\right) \mid \mathcal{G}_{t}\right] \\
& \leq \mathbb{E}\left[\mathbb{P}\left(\mathrm{S} \in \mathcal{U} \mid \Im_{0}\right) \mid \mathcal{G}_{t}\right] \\
& =\mathbb{E}\left[\mathbb{I P}\left(\mathrm{S} \in \mathcal{U} \mid \mathcal{G}_{0}\right) \mid \mathcal{G}_{t}\right] \\
& =\mathbb{P}\left(\mathrm{S} \in \mathcal{U} \mid \mathcal{G}_{0}\right) .
\end{aligned}
$$

Uma outra propriedade adicional à classe $M N B U \mid \Im_{t}$ é dada pelo seguinte teorema:

Teorema 1.2.3: Suponhamos que $\mathbf{S}=\left(S_{i}\right)_{1 \leq i \leq n}$ é $M N B U \mid \Im_{t}$, onde $\left(\Im_{t}\right)_{t \geq 0}$ é a família de $\sigma$-álgebras gerada pelos indicadores dos componentes. Se $I_{0}$ é um subconjunto de $\{1, \ldots, n\}, \mathbf{S}_{0}=\left(S_{i}\right)_{i \in I_{0}}$ e $\mathcal{G}_{t}$ é a família de $\sigma$-álgebras geradas pelos indicadores dos componentes $\left(S_{i}\right)_{i \in I_{0}}$, então $\mathbf{S}_{0}$ é $M N B U \mid \mathcal{G}_{t}$. Em particular, cada $S_{i}$ é $N B U$.

Prova: Primeiramente observemos que um conjunto superior $\mathcal{U}_{0}$ em $\mathbb{R}^{\operatorname{card}\left(I_{0}\right)}$ pode ser escrito como $\mathcal{U}=\left\{\mathrm{x} \in \mathbb{R}^{n}:\left(x_{i}\right)_{i \in I_{0}} \in \mathcal{U}_{0}\right\}$ e portanto para todo $t \geq 0$,

$$
\begin{aligned}
\mathbb{P}\left(\theta_{t} \mathrm{~S}_{0} \in \mathcal{U}_{0} \mid \Im_{t}\right) & =\mathbb{P}\left(\theta_{t} \mathbf{S} \in \mathcal{U} \mid \Im_{t}\right) \\
& \leq \mathbb{P}\left(\mathbf{S} \in \mathcal{U} \mid \Im_{0}\right) \\
& =\mathbb{P}\left(\mathbf{S}_{0} \in \mathcal{U}_{0} \mid \Im_{0}\right)
\end{aligned}
$$

e então $\mathrm{S}_{0}$ é $M N B U \mid \Im_{t}$. Utilizando o Teorema 1.2 .2 , concluímos que $\mathrm{S}_{0}$ é $M N B U \mid \mathcal{G}_{t}$.

Notemos que o resultado acima só é válido na classe de distribuições $M I F R \mid \Im_{t}$ na presença da independência entre os vetores $\mathbf{S}_{\mathbf{0}}=\left(S_{i}\right)_{i \in I_{0}}$ e $\mathbf{S}_{\mathbf{0}}^{\mathbf{c}}=\left(S_{i}\right)_{i \in I_{0}^{c}}$.

No caso clássico, a classe das distribuições $N B U$ é fechada por formação de sistemas monótonos. Esta propriedade é preservada na classe $M N B U \mid \Im_{t}$ : 
Teorema 1.2.4: Suponhamos que $\mathrm{S}$ seja $M N B U \mid \Im_{t}$ onde $\Im_{t}$ é a $\sigma$-álgebra gerada pelos indicadores dos componentes $S_{i}, 1 \leq i \leq n$. Se $\tau$ é o tempo de vida de um sistema monótono e $\mathcal{G}_{t}=\sigma\left\{1_{\{\tau>s\}}: s \leq t\right\}$, então $\tau$ é $N B U$.

Prova: Como na prova do Teorema 1.1.4, para $f: \mathbb{R} \longrightarrow \mathbb{R}$ crescente, temos que

$$
f\left(\theta_{t} \tau\right)=f\left(\tau\left(\theta_{t} \mathbf{S}\right)\right)=(f \circ \tau)\left(\theta_{t} \mathbf{S}\right)
$$

onde a função composta $f \circ \tau: \mathbb{R}^{n} \longrightarrow \mathbb{R}$ é crescente. Como S é $M N B U \mid \Im_{t}$, temos que

$$
\mathbb{E}\left[(f \circ \tau)\left(\theta_{t}(\mathbf{S}) \mid \Im_{t}\right] \leq \mathbb{E}\left[(f \circ \tau)(\mathbf{S}) \mid \Im_{0}\right]\right.
$$

e portanto

$$
\mathbb{E}\left[f\left(\theta_{t} \tau\right) \mid \Im_{t}\right] \leq \mathbb{E}\left[f(\tau) \mid \Im_{0}\right]
$$

isto é, $\tau$ é $N B U \mid \Im_{t}$. Como $\mathcal{G}_{t} \subset \Im_{t}$ para todo $t>0$ e $\mathcal{G}_{0}=\Im_{0}$, utilizando o Teorema 1.2.2, concluímos que $\tau$ é $N B U$. 
Cap. 1: Classes de distribuições de vida condicionadas à $\sigma$-álgebra do passado observado

\subsection{Caracterização de classes de distribuições através do processo de risco.}

Seja $\tau$ o tempo de vida de um objeto e $F(t)=\mathbb{P}(\tau \leq t)$ sua função de distribuição. Considere a função definida pela integral de Stieltjes:

$$
R(t)=\int_{(0, t]} \frac{F(d s)}{F[s, \infty)}
$$

onde $F[s, \infty)=\mathbb{P}(\tau \geq s)$.

Vejamos algumas propriedades de $R(t)$ :

1- $R(0)=0$ e $R(t)$ é uma função não decrescente, pois o integrando é positivo.

2- $R(t)$ é contínua à direita, pois considerando-se uma sequência $\left(t_{n}\right)_{n \geq 1} \operatorname{com} t_{n} \downarrow t$ temos que

$$
\begin{aligned}
(0, t] & =\bigcap_{n=1}^{\infty}\left(0, t_{n}\right] \mathrm{e} \\
\int_{(0, t]} \frac{F(d s)}{F[s, \infty)} & =\lim _{n \rightarrow \infty} \int_{\left(0, t_{n}\right]} \frac{F(d s)}{F[s, \infty)} .
\end{aligned}
$$

3- $R(t)$ tem os mesmos pontos de descontinuidade de $F$, com

$$
\Delta R(t)=R(t)-R\left(t^{-}\right)=\frac{\mathbb{P}(\tau=t)}{F[t, \infty)} \leq 1
$$

4- No caso em que $\Delta R(t)=1$ para algum $t$, temos $\mathbb{I P}(\tau>t)=0$ e $R(s)=R(t)$, para $s \geq t$. 
Como mostra o próximo teorema, a classe das funções com as propriedades acima caracteriza as medidas de probabilidade em $(0, \infty]$.

Teorema 1.3.1: Seja $\aleph$ a classe de todas as funções $R(t)$ que são não decrescentes, contínuas à direita, com $R(0)=0, \Delta R(t) \leq 1$ e $R(s)=R(t)$, para $s \geq t$ quando $\Delta R(t)=1$. Então

$$
F(0, t]=1-\exp \left\{-R_{c}(t)\right\} \prod_{s \leq t}(1-\Delta R(s))
$$

onde $R_{c}(t)$ é a parte contínua de $R(t)$ e

$$
R(t)=\int_{(0, t]} \frac{F(d s)}{F[s, \infty)}
$$

definem uma correspondência biunívoca entre a classe de todas as medidas de probabilidade em $(0, \infty)$ e a classe $\aleph$.

Prova: Para cada medida $F(0, t]$ podemos definir $R(t)=\int_{(0, t]} \frac{F(d s)}{F[s, \infty)}$ e inversamente, para cada $R(t)$ em $\aleph$, podemos definir a medida $F(0, t]=1-\exp \left\{-R_{c}(t)\right\} \prod_{s \leq t}(1-$ $\Delta R(s))$. Suponhamos que existam duas medidas de probabilidades $F_{1}(0, t]$ e $F_{2}(0, t]$ tais que

$$
R(t)=\int_{(0, t]} \frac{F_{1}(d s)}{F_{1}[s, \infty)}=\int_{(0, t]} \frac{F_{2}(d s)}{F_{2}[s, \infty)}
$$

Diferenciando as equações em $t$ e integrando convenientemente, temos:

$$
\begin{gathered}
1-\int_{0}^{t} F_{1}[s, \infty) R(d s)=F_{1}[t, \infty) \quad \mathrm{e} \\
1-\int_{0}^{t} F_{2}[s, \infty) R(d s)=F_{2}[t, \infty),
\end{gathered}
$$


$\operatorname{assim} F_{1}$ e $F_{2}$ satisfazem a equação $1-\int_{0}^{t} F[s, \infty) R(d s)=F[t, \infty)$ que pelo Lema 18.8 Liptser \& Shiryayev (1977b) tem solução única e igual a

$$
F_{1}[t, \infty)=F_{2}[t, \infty)=\exp \left\{-R_{c}(t)\right\} \prod_{s \leq t}(1-\Delta R(s))
$$

Logo a correspondência é bijetora e o teorema está demonstrado.

No caso em que a função de distribuição de $\tau, F(t)=F(0, t]$ é contínua, essa relação de equivalência reduz-se a

$$
R(t)=-\log \bar{F}(t)
$$

onde $\bar{F}(t)=1-F(t)$.

Nesta forma, $R(t)$ é frequentemente utilizada para caracterizar classes de distribuições de vida úteis na Teoria da Clássica da Confiabilidade, como por exemplo:

a) Uma função de distribuição $F$ pertence à classe de distribuição de vida com taxa de falha crescente $(I F R)$ se e somente se $R(t)$ é convexa.

b) Uma função de distribuição $F$ pertence à classe das distribuições $N B U$ se e somente se $R(t+s) \geq R(t)+R(s)$, para todo $s$ e $t \geq 0$.

Com essas caracterizações , $R(t)=-\log \bar{F}(t)$ é frequentemente definida como função acumulada de risco e isso não corresponde à realidade pois, no caso geral, a distribuição pode ter uma parte discreta

$$
F(0, t]=1-\exp \left\{-R_{c}(t)\right\} \prod_{s \leq t}(1-\Delta R(s))
$$


Reservando-nos contra esse inconveniente, adotaremos a seguinte definição :

Definição 1.3.2: Seja $\tau$ o tempo de vida de um objeto com função de distribuição $F$. Então

$$
R(t)=\int_{(0, t]} \frac{F(d s)}{F[s, \infty)}
$$

é denominada função de risco de $\tau$ (ou de $F$ ).

Com a definição acima preservamos a noção intuitiva de uma função de risco, ou seja,

$$
R(d t)=\frac{F(d t)}{F[t, \infty)}=\mathbb{P}(\tau \in d t \mid \tau \geq t)
$$

Consideremos $\tau$, o tempo de vida de um objeto, o seu indicador de falha $N(t)=$ $1_{\{\tau \leq t\}}$ e a família de $\sigma$-álgebras $\left(\Im_{t}\right)_{t \geq 0}, \Im_{t}=\sigma\left\{1_{\{\tau>s\}}: s \leq t\right\}$, crescentes, contínuas à direita e completas. Claramente, $(N(t))_{t \geq 0}$ é um submartingale e podemos concluir, utilizando a decomposição de Doob-Meyer, que existe um martingale $(M(t))_{t \geq 0}$ e um único processo natural crescente $(A(t))_{t \geq 0}(\operatorname{com} A(0)=0)$, o $\Im_{t}$-compensador de $(N(t))_{t \geq 0}$, tal que

$$
N(t)=M(t)+A(t), \quad t \geq 0
$$

Proposição 1.3.3: Sob as condições acima,

$$
A(t)=\int_{0}^{t \wedge \tau} \frac{F(d s)}{F[s, \infty)}
$$

Prova: Ver Liptser e Shiryayev (1977a) 
O fato importante a ser considerado é que o $\Im_{t}$-compensador

$$
A(t)=\int_{(0, t \wedge \tau]} \frac{F(d s)}{F[s, \infty)}
$$

é a nossa função de risco no conjunto de interesse $\{t \leq \tau\}$ e, caracteriza as distribuições $I F R$ e $N B U$ no caso contínuo univariado.

Observe que os compensadores são determinados relativamente a uma família de $\sigma$ álgebras $\left(\Im_{t}\right)_{t \geq 0}$ fixada. Se mudarmos esta família mudaremos o compensador. Portanto, ao caracterizar uma distribuição através dos compensadores devemos considerar a família de $\sigma$-álgebras envolvida.

A seguir generalizaremos a noção de risco através dos compensadores.

Em um sistema $\phi$ as falhas dos componentes $C_{1}, \ldots, C_{n}$ aparecem no decorrer do tempo como um processo estocástico e este fato é interpretado convenientemente através de um processo pontual multivariado. Para determinada realização $\omega$ fixada, seja $q(\omega)$ o número de valores distintos no conjunto $\left\{S_{i}(\omega), 1 \leq i \leq n\right\}$, as estatísticas de ordem crescentes desse conjunto denotadas por

$$
T_{1}(\omega)<T_{2}(\omega)<\ldots<T_{q(\omega)}(\omega)
$$

e definiremos também o conjunto dos componentes que falham no $k$-ésimo tempo de falha $T_{k}$, por

$$
X_{k}(\omega)=\left\{i: 1 \leq i \leq n, S_{i}(\omega)=T_{k}(\omega)\right\}
$$

Se não existirem falhas múltiplas, o valor de $X_{k}$ é um dos conjuntos $\{i\}, 1 \leq i \leq n$, mas em geral, $X_{k}$ é uma variável aleatória com valores em $\Lambda=\mathcal{P}\{1,2, \ldots, n\}$, o conjunto das partes de $\{1,2, \ldots, n\}$. 
Chamaremos $T_{k}$ o $k$-ésimo tempo de falha e $X_{k}$ o $k$-ésimo conjunto de falha. Assim, a sequência aleatória $\left(T_{k}, X_{k}\right)_{1 \leq k \leq q}$ descreve como os componentes $C_{1}, \ldots, C_{n}$ falharam e considerando $T_{q+1}=T_{q+2}=\ldots=\infty$ e $X_{q+1}=X_{q+2}=\ldots=\emptyset$ podemos definir:

Definição 1.3.3: Definimos o Processo de Falhas dos Componentes como o processo pontual marcado $\left(T_{k}, X_{k}\right)_{k \geq 1}$.

Uma maneira equivalente de descrever esse processo é através dos processos de contagem: para cada conjunto de falha $I \in \Lambda$ fixado, sejam $\tau_{I}$ e $N_{I}(\omega ; t)$ definidos por

$$
\begin{gathered}
\tau_{I}=\inf \left\{T_{k}: X_{k}=I\right\} \quad(\inf \emptyset=\infty) \quad e \\
N_{I}(\omega ; t)= \begin{cases}0, & \text { se } t<\tau_{I}(\omega), \\
1, & \text { se } t \geq \tau_{I}(\omega) .\end{cases}
\end{gathered}
$$

Para cada $\omega,\left(S_{i}(\omega)\right)_{1 \leq i \leq n}$ determina a realização do processo $\left(N_{I}(\omega, t) ; I \in \Lambda\right)_{t \geq 0}$ e vice-versa. Similarmente

$$
\Im_{t}=\sigma\left\{1_{\left\{S_{i} \leq s\right\}} ; s \leq t, 1 \leq i \leq n\right\} \text { é equivalente a } \Im_{t}=\sigma\left\{N_{I}(s) ; s \leq t, I \in \Lambda\right\} .
$$

Analisaremos a noção de risco através dos compensadores do processo pontual multivariado $\left(N_{I}(t) ; I \in \Lambda\right)_{t \geq 0}$. Começaremos com um conjunto de componentes fixado $I \in \Lambda$, e o processo de contagem $\left(N_{I}(t)\right)_{t \geq 0}$ associado a $I$.

Notemos que $\left(N_{I}(t)\right)_{t \geq 0}$ admite a decomposição de Doob-Meyer

$$
N_{I}(t)=M_{I}(t)+A_{I}(t),
$$

onde $\left(M_{I}(t)\right)_{t \geq 0}$ é um $\Im_{t}$-martingale uniformemente integrável, $\left(A_{I}(t)\right)_{t \geq 0}$ é um processo previsível crescente e $\Im_{t}=\sigma\left\{N_{I}(s), s \leq t, I \in \Lambda\right\}$. 
Definição 1.3.4: A família de processos

$$
\left(A_{I}(t) ; I \in \Lambda\right)_{t \geq 0}
$$

indexado por $I \in \Lambda$ é denominada processo de risco multivariado.

Teorema 1.3.5: Nas condições acima, $A_{I}(t)$ pode ser expresso como

$$
A_{I}(d t)=\sum_{k \geq 0} \frac{G_{k}\left(d t-T_{k}, I\right)}{H_{k}\left(\left[t-T_{k}, \infty\right]\right)} 1_{\left\{T_{k}<t \leq T_{k+1}\right\}}, \quad \text { q.c. }
$$

onde $G_{k}(d s, I)$ é uma versão regular da probabilidade condicional de $\left(T_{k+1}, X_{k+1}\right)$, dado $\Im_{T_{k}}$ e $H_{k}(d t)=G_{k}(d t, \Lambda)$ é a distribuição condicional de $T_{k+1}-T_{k}$ dado $\Im_{T_{k}}$.

A prova deste teorema é encontrada em Arizono (1992).

Observemos que o $k$-ésimo termo de $d A_{I}(t)$ assume um valor não nulo somente no intervalo aleatório $\left(T_{k}, T_{k+1}\right]$ e, neste intervalo, ele expressa a probabilidade condicional de $\left\{T_{k+1} \in d t, X_{k+1}=I\right\}$, dados os tempos de falhas precedentes $T_{1}, \ldots, T_{k}$, seus respectivos conjuntos de falhas $X_{1}, \ldots, X_{k}$, e $T_{k+1} \geq t$. Neste sentido, $\left(A_{I}(t): t \geq 0, I \in \Lambda\right)$ expressa somente os riscos que são imediatos ou associados com a próxima falha.

No caso clássico univariado sabemos que se $\tau$ é o tempo de vida de um objeto, com função de distribuição $F$, absolutamente contínua, a função de risco $R(t)$ caracteriza as classes de distribuições $I F R$ e $N B U$.

No que segue, analisaremos as relações entre a realização do Processo de Risco e as classes $I F R \mid \Im_{t}$ e $N B U \mid \Im_{t}$, do tempo de vida do sistema. Para tanto usaremos a calculabilidade dos compensadores (diremos que o $\left(\Im_{t}\right)$-compensador $A_{I}(t)$, onde $\Im_{t}=$ $\sigma\left\{N_{I}(s), s \leq t, I \in \Lambda\right\}$, é calculável se é o limite em probabilidade de uma soma de probabilidades condicionais. 
Definição 1.3.6: Definiremos $\left(Q_{n}\right)_{n \geq 1}$, uma $R$-sequência de partições do intervalo $(0, s]$ como uma sequência de partições tal que:

(1) Se $n \geq m$ então $Q_{n}$ é um refinamento de $Q_{m}$.

(2) $\max _{\left(t, t^{*}\right] \in Q_{n}}\left(t^{*}-t\right) \rightarrow 0$.

Definição 1.3.7: Seja $a\left(Q_{n}\right)=\sum_{\left(t, t^{*}\right] \in Q_{n}} \mathbb{E}\left[N_{I}\left(t^{*}\right)-N_{I}(t) \mid \Im_{t}\right]$. Dizemos que $A_{I}$ é calculável se, para qualquer $s \geq 0$ e qualquer $R$-sequência de partições $\left(Q_{n}\right)_{n \geq 1}$ de $(0, s]$, tivermos

$$
a\left(Q_{n}\right) \rightarrow A_{I}(s)
$$

em probabilidade.

Definição 1.3.8: Dizemos que $A$ é calculável localmente (L-calculável) se o compensador $A\left(t \wedge T_{k}\right)$ de $N\left(t \wedge T_{k}\right)$ é calculável para cada $k$.

Teorema 1.3.9: Seja $\Im_{t}=\sigma\left\{N_{I}(s), s \leq t, I \in \Lambda\right\}$. O $\Im_{t}$-compensador $A_{I}(t)$ de $N_{I}(t)$ é L-calculável.

Para provar a proposição acima devemos usar os dois seguintes lemas

Lema 1.3.10: Se $\mathcal{G}$ e $\mathcal{H}$ são duas sub- $\sigma$-álgebras de $\Im, B$ é um evento arbitrário, $C \in \mathcal{G}$ e $\mathcal{G} \cap C=\mathcal{H} \cap C, \operatorname{com} \mathcal{G} \cap C=\{A \cap C: A \in \mathcal{G}\}$ e $\mathcal{H} \cap C=\{A \cap C: A \in \mathcal{H}\}$, então

$$
\mathbb{P}(B \cap C \mid \mathcal{G})= \begin{cases}\frac{\mathbb{P}(B \cap C \mid \mathcal{H})}{\mathbb{P}(C \mid \mathcal{H})}, & \text { se } \omega \in C \\ 0, & \text { se } \omega \in C^{c}\end{cases}
$$

onde $\mathbb{P}(C \mid \mathcal{H})$ é uma versão para a qual $\mathbb{P}(C \mid \mathcal{H})>0$ em $C$. 
Prova: Seja $D \in \mathcal{G}$

$$
\begin{aligned}
\int_{D} \mathbb{P}\{B \cap C \mid \mathcal{G}) \mathbb{P}(d \omega) & \left.=\int_{D \cap C} \mathbb{P}(B \cap C \mid \mathcal{G}) \mathbb{P}(d \omega)+\int_{D \cap C^{c}} \mathbb{P}(B \cap C) \mid \mathcal{G}\right) \mathbb{P}(d \omega) \\
& =\int_{D \cap C} \frac{\mathbb{P}(B \cap C \mid \mathcal{H})}{\mathbb{P}(C \mid \mathcal{H})} \mathbb{P}(d \omega) \\
& =\int_{D \cap C} \mathbb{P}(B \cap C \mid C \cap \mathcal{H}) \mathbb{P}(d \omega) \\
& =\int_{D \cap C} \mathbb{P}(B \cap C \mid C \cap \mathcal{G}) \mathbb{P}(d \omega) \\
& =\mathbb{P}(B \cap C \cap D) .
\end{aligned}
$$

Lema 1.3.11: Para todo $k \geq 0$, e todo $t^{*}>t \geq 0$ temos

$$
\begin{aligned}
& \mathbb{P}\left(\left\{T_{k}<t<T_{k+1} \leq t^{*}\right\} \cap\left\{X_{k+1}=I\right\} \mid \Im_{t}\right)= \\
& = \begin{cases}\frac{\mathbb{P}\left(\left\{t<T_{k+1} \leq t^{*}\right\} \cap\left\{I=X_{k+1}\right\} \mid \Im_{T_{k}}\right)}{\mathbb{P}\left(t<T_{k+1} \mid \Im_{T_{k}}\right)}, & \text { se } \omega \in\left\{T_{k}<t<T_{k+1}\right\} \\
0, & \text { c.c. }\end{cases}
\end{aligned}
$$

onde $\operatorname{IP}\left(t<T_{k+1} \mid \Im_{T_{k}}\right)$ é uma versão não nula no conjunto $\left\{T_{k}<t<T_{k+1}\right\}$.

Prova: Considere $C=\left\{T_{k}<t<T_{k+1}\right\} \in \Im_{t}$. Note também que

$$
\left\{\left\{N_{I}(t)=r\right\} \cap\left\{T_{k}<t<T_{k+1}\right\}\right\}=\left\{\left\{N_{I}\left(T_{k}\right)=r\right\} \cap\left\{T_{k}<t<T_{k+1}\right\}\right\}
$$

de forma que $C \cap \Im_{t}=C \cap \Im_{T_{k}}$.

Podemos então considerar $\mathcal{G}=\Im_{t}, \mathcal{H}=\Im_{T_{k}}, B=\left\{T_{k+1} \leq t^{*}\right\} \cap\left\{X_{k+1}=I\right\}$ e $C=\left\{T_{k}<t<T_{k+1}\right\}$ e desde que $C \in \Im_{t}$, aplicar o Lema 1.3.10. 
Prova:(Teorema 1.3.9). Sabemos que

$$
A_{I}(t)=\sum_{k \geq 0} \int_{T_{k}}^{t \wedge T_{k+1}} \frac{G_{k}(d u, I)}{H_{k}[u, \infty)}
$$

Consideremos para cada $k \geq 0$ o compensador:

$A_{I}^{k}(s)=A_{I}\left(s \wedge T_{k+1}\right)-A_{I}\left(s \wedge T_{k}\right) \operatorname{de} N_{I}^{k}(s)=N_{I}\left(s \wedge T_{k+1}\right)-N_{I}\left(s \wedge T_{k}\right)$

Seja $\left(Q_{n}\right)_{n \geq 1}$ uma $R$-sequência de partições de $(0, s]$. Se $t, t^{*} \in \mathbb{R}_{+}$, temos

$$
N_{I}^{k}\left(t^{*}\right)-N_{I}^{k}(t)=I_{\left\{t<T_{k+1} \leq t^{*}\right\}} I_{\left\{X_{k+1}=I\right\}}
$$

Assim,

$$
\begin{aligned}
a\left(Q_{n}\right)= & \sum_{\left(t, t^{*}\right] \in Q_{n}} \mathbb{E}\left(N_{I}^{k}\left(t^{*}\right)-N_{I}^{k}(t) \mid \Im_{t}\right) \\
= & \sum_{\left(t, t^{*}\right] \in Q_{n}} \mathbb{E}\left(I_{\left\{t<T_{k+1} \leq t^{*}\right\}} I_{\left\{X_{(k+1)}=I\right\}} \mid \Im_{t}\right) \\
= & \sum_{\left(t, t^{*}\right] \in Q_{n}} \mathbb{E}\left(I_{\left\{t \leq T_{k}<T_{k+1} \leq t^{*}\right\}} I_{\left\{X_{k+1}=I\right\}} \mid \Im_{t}\right)+ \\
& +\sum_{\left(t, t^{*}\right] \in Q_{n}} \mathbb{E}\left(I_{\left\{T_{k}<t<T_{k+1} \leq t^{*}\right\}} I_{\left\{X_{k+1}=I\right\}} \mid \Im_{t}\right)
\end{aligned}
$$


Como

$$
\begin{aligned}
& \left\|\sum_{\left(t, t^{*}\right] \in Q_{n}} \mathbb{E}\left(I_{\left\{t \leq T_{k}<T_{k+1} \leq t^{*}\right\}} I_{\left\{X_{k+1}=I\right\}} \mid \Im_{t}\right)\right\|_{1}= \\
& =\sum_{\left(t, t^{*}\right] \in Q_{n}} \mathbb{P}\left(t \leq T_{k}<T_{k+1} \leq t^{*}, X_{k+1}=I\right) \rightarrow 0
\end{aligned}
$$

quando $n \rightarrow \infty$ temos

$$
a\left(Q_{n}\right) \sim \sum_{\left(t, t^{*}\right] \in Q_{n}} \mathbb{P}\left(T_{k}<t<T_{k+1} \leq t^{*}, X_{k+1}=I \mid \Im_{t}\right)
$$

Utilizando o Lema 1.3.11 concluímos que em $\left(T_{k}<t<T_{k+1}\right)$

$$
a\left(Q_{n}\right) \sim \sum_{\left(t, t^{*}\right] \in Q_{n}} \frac{\mathbb{P}\left(\left\{t<T_{k+1} \leq t^{*}\right\} \cap\left\{X_{k+1}=I\right\} \mid \Im_{T_{k}}\right)}{\mathbb{P}\left(t<T_{k+1} \mid \Im_{T_{k}}\right)}
$$

Para cada $\omega \in\left\{T_{k}<s\right\}$ podemos reconhecer que cada termo da soma do lado direito de (1.3.11) é a integral da função $\sum_{\left(t, t^{*}\right] \in Q_{n}} I_{\left(t, t^{*}\right]}(u) \frac{1}{H_{k}\left[t^{*}, \infty\right)}$ com respeito à medida $G_{k}(d u, I)$, e concluímos, aplicando o teorema de convergência monótona para estas funções escadas, que para cada $\omega_{0} \in\left\{T_{k}<s\right\}$ obtemos

$$
a\left(Q_{n}\right) \rightarrow \sum_{k \geq 0} \int_{T_{k}}^{s \wedge T_{k+1}} \frac{G_{k}(d u, I)}{H_{k}[u, \infty)}=A_{I}(s)
$$


Cap. 1: Classes de distribuições de vida condicionadas à $\sigma$-álgebra do passado observado

$\operatorname{Assim} \mathbb{P}\left(\left\{a\left(Q_{n}\right) \nrightarrow A_{I}(s)\right\} \cap\left\{T_{k}<s\right\}\right)=0$, e como $\Omega=\cup\left\{T_{k}<s\right\}$ temos que

$$
\begin{aligned}
\mathbb{P}\left(\left\{a\left(Q_{n}\right) \nrightarrow A_{I}(s)\right\} \cap \Omega\right) & =\mathbb{P}\left(\left\{a\left(Q_{n}\right) \nrightarrow A_{I}(s)\right\} \cap \cup_{k}\left\{T_{k}<s\right\}\right) \\
& =\mathbb{P}\left(\cup_{k}\left\{\left\{a\left(Q_{n}\right) \nrightarrow A_{I}(s)\right\} \cap\left\{T_{k}<s\right\}\right\}\right) \\
& \leq \sum_{k} \mathbb{P}\left(\left\{a\left(Q_{n}\right) \nrightarrow A_{I}(s)\right\} \cap\left\{T_{k}<s\right\}\right) \\
& =0
\end{aligned}
$$

portanto, $\left\{a\left(Q_{n}\right) \rightarrow A_{I}(s)\right\}$ em probabilidade.

Corolário 1.3.12: Em probabilidade, a seguinte convergência é válida

$$
\sum_{\frac{k}{2^{n}}<t} \mathbb{P}\left\{\frac{k}{2^{n}}<\tau_{\phi} \leq \frac{k+1}{2^{n}} \mid \Im_{\frac{k}{2^{n}}}\right\} \rightarrow A_{\phi}(t)
$$

\section{Prova:}

Seja $\sigma_{\phi}(I)$ o instante a partir do qual o conjunto de falhas $I$ se torna crítico para o sistema, então

$$
\begin{aligned}
\sum_{\frac{k}{2^{n}}<t} \mathbb{P}\left\{\frac{k}{2^{n}}<\tau \leq \frac{k+1}{2^{n}} \mid \Im_{\frac{k}{2^{n}}}\right\} & =\sum_{\frac{k}{2^{n}}<t} \sum_{I \in \Lambda} 1_{\left\{\sigma_{\phi}(I)<\frac{k}{2^{n}}<\tau \wedge t\right\}} \mathbb{I P}\left\{\frac{k}{2^{n}}<\tau_{I} \leq \frac{k+1}{2^{n}} \mid \Im_{\frac{k}{2^{n}}}\right\} \\
& =\sum_{I \in \Lambda} \sum_{\sigma_{\phi}(I)<\frac{k}{2^{n}}<\tau \wedge t} \mathbb{P}\left\{\frac{k}{2^{n}}<\tau_{I} \leq \frac{k+1}{2^{n}} \mid \Im_{\frac{k}{2^{n}}}\right\} \\
& \rightarrow \sum_{I \in \Lambda}\left[A_{I}(t \wedge \tau)-A_{I}\left(\sigma_{\phi}\right)\right]^{+} \\
& =A_{\phi}(t) .
\end{aligned}
$$


Teorema 1.3.13: Suponhamos que $\phi$ seja um sistema monótono com tempo de vida $\tau$ que é $I F R \mid \Im_{t}$. Então as realizações do Processo de Risco $\left(A_{\phi}(t)\right)_{t \geq 0}$ são convexas q.c. em $t \in(0, \tau]$.

Prova: Seja $Q=\left\{\frac{k}{2^{n}}: k, n \in N\right\}$. Como $A_{\phi}(t)$ é contínuo à direita e $Q$ é denso em $R^{+}$, é suficiente provar que

$$
A_{\phi}(\omega ; t+h)-A_{\phi}(\omega ; t) \geq A_{\phi}\left(\omega ; t^{*}+h\right)-A_{\phi}\left(\omega ; t^{*}\right)
$$

para todo $t, t^{*}$ e $h \in Q$ tais que $0 \leq t^{*} \leq t+h \leq \tau(\omega)$, pois neste caso, $A_{\phi}(\omega ; t)$ tem derivada crescente e portanto é convexa em $(0, \tau(\omega)$ ) (a convexidade em $\tau(\omega)$ segue desde que $A_{\phi}(t)$ é crescente e contínua à direita).

Para provar a desigualdade acima usaremos que o compensador $A_{\phi}(t)$ é calculável (Brown (1978)). Esta propriedade assegura que $B_{n}(t) \rightarrow A_{\phi}(t)$ em probabilidade quando $n \rightarrow \infty$ onde $B_{n}(t)=\sum_{\frac{j}{2^{n}}<t} \mathbb{P}\left(\frac{j}{2^{n}}<\tau \leq \frac{j+1}{2^{n}} \mid \Im_{\frac{j}{2^{n}}}\right)$.

Se $I P^{*}\left(\frac{j}{2^{n}}<\tau \leq \frac{j+1}{2^{n}} \mid \Im_{\frac{j}{2^{n}}}\right)$ é uma versão regular de $\mathbb{I P}\left(\frac{j}{2^{n}}<\tau \leq \frac{j+1}{2^{n}} \mid \Im_{\frac{j}{2^{n}}}\right)$ e $B_{n}^{*}(t)$ é a respectiva versão de $B_{n}(t)$, existe um conjunto de medida nula $N_{0}$ e uma subsequência $\left(B_{n_{k}}^{*}(t)\right)_{k \geq 1}$, tal que se $\omega \notin N_{0}, B_{n_{k}}^{*}(\omega ; t) \rightarrow A_{\phi}(\omega ; t)$ quando $k \rightarrow \infty$. Consequentemente, para $\omega \notin N_{0}$ e $t, h \in Q$ vale:

$$
\begin{aligned}
A_{\phi}(\omega ; t+h)-A_{\phi}(\omega ; t) & =\lim _{k \rightarrow \infty}\left(B_{n_{k}}^{*}(\omega ; t+h)-B_{n_{k}}^{*}(\omega ; t)\right) \\
& =\lim _{k \rightarrow \infty} \sum_{0 \leq \frac{j}{2^{n_{k}}}<h} I P^{*}\left(t+\frac{j}{2^{n_{k}}}<\tau \leq t+\frac{j+1}{2^{n_{k}}} \mid \Im_{t+\frac{j}{2^{n_{k}}}}\right) .
\end{aligned}
$$


Contudo $\tau$ é $I F R \mid \Im_{t}$ e assim, no conjunto $\left\{t+\frac{j}{2^{n_{k}}}<\tau\right\}$ temos

$$
\begin{aligned}
\mathbb{P}^{*}\left(t+\frac{j}{2^{n_{k}}}<\tau \leq t+\frac{j+1}{2^{n_{k}}} \mid \Im_{t+\frac{j}{2^{n_{k}}}}\right) & =1-\mathbb{P}^{*}\left(\left(\tau-t-\frac{j}{2^{n_{k}}}\right)^{+}>\frac{1}{2^{n_{k}}} \mid \Im_{t+\frac{j}{2^{n_{k}}}}\right) \\
& \geq 1-\mathbb{P}^{*}\left(\left(\tau-t^{*}-\frac{j}{2^{n_{k}}}\right)^{+}>\frac{1}{2^{n_{k}}} \mid \Im_{t^{*}+\frac{j}{2^{n_{k}}}}\right) \\
& =\mathbb{I}^{*}\left(t^{*}+\frac{j}{2^{n_{k}}}<\tau \leq t^{*}+\frac{j+1}{2^{n_{k}}} \mid \Im_{t^{*}+\frac{j}{2^{n_{k}}}}\right) \quad \text { q.c. }
\end{aligned}
$$

Desta maneira, existe um conjunto de medida nula $N_{k}$ tal que se $\omega \notin N_{k}$, vale

$$
\begin{aligned}
& \sum_{0 \leq \frac{j}{2^{n_{k}}}<h} \mathbb{P}^{*}\left(t+\frac{j}{2^{n_{k}}}<\tau \leq t+\frac{j+1}{2^{n_{k}}} \mid \Im_{t+\frac{j}{2^{n_{k}}}}\right) \\
\geq & \sum_{0 \leq \frac{j}{2^{n_{k}}}<h} \mathbb{P}^{*}\left(t^{*}+\frac{j}{2^{n_{k}}}<\tau \leq t^{*}+\frac{j+1}{2^{n_{k}}} \mid \Im_{t^{*}+\frac{j}{2^{n_{k}}}}\right)
\end{aligned}
$$

para todo $t, t^{*}$ e $h \in Q$ tais que $0 \leq t^{*} \leq t+h \leq \tau(\omega)$. Assim, se $\omega$ não pertence ao conjunto nulo $N=N_{0} \bigcup\left(\bigcup_{k} N_{k}\right)$, concluímos que

$$
A_{\phi}(\omega ; t+h)-A_{\phi}(\omega ; t) \geq A_{\phi}\left(\omega ; t^{*}+h\right)-A_{\phi}\left(\omega ; t^{*}\right)
$$

como queríamos demonstrar.

A recíproca do teorema acima não é verdadeira, como mostra o seguinte contraexemplo: 
Exemplo 1.3.14: Consideremos um sistema monótono com tempo de vida $\tau$ com componentes $C_{1}$ e $C_{2}$. O componente $C_{1}$ tem tempo de vida $S_{1}$ com distribuição exponencial de parâmetro 1 e o componente $C_{2}$ tem o tempo de vida $S_{2}=S_{1}+Y$, com

$$
\mathbb{I P}\left(Y \leq y \mid S_{1}\right)=1-\exp \left\{-\alpha\left(S_{1}\right) y\right\}
$$

onde a função $\alpha(t)$ é estritamente decrescente e positiva. O tempo de vida do sistema é dado por $\tau=S_{2}=S_{1}+Y$.

Arizono (1992) prova que uma forma explícita para o compensador $A_{\phi}(t)$ é dado por

$$
A_{t}(\phi)=\sum_{I \in \Lambda}\left[A_{t \wedge \tau}(I)-A_{\sigma_{\phi}(I)}(I)\right]^{+} \text {q.c. }
$$

onde $\sigma_{\phi}(I), I \in \Lambda$ é um tempo de parada, instante a partir do qual o conjunto de falhas $I$ se torna crítico para o sistema $\phi$.

Como $S_{1}$ nunca é crítica, temos que $\sigma_{\phi}(1)=\infty$ e desde que $Y \mid S_{1}$ é exponencial de parâmetro $\alpha\left(S_{1}\right)$, concluímos que $\sigma_{\phi}(2)=S_{1}$. Assim,

$$
\begin{aligned}
A_{\phi}(t) & =\left[A_{1}(t \wedge \tau)-A_{1}\left(\sigma_{\phi}(1)\right]^{+}+\left[A_{2}(t \wedge \tau)-A_{2}\left(\sigma_{\phi}(2)\right)\right]^{+}\right. \\
& =\left[A_{1}(t \wedge \tau)-A_{1}(\infty)\right]^{+}+\left[A_{2}(t \wedge \tau)-A_{2}\left(S_{1}\right)\right]^{+} \\
& =\left[A_{2}(t \wedge \tau)-A_{2}\left(S_{1}\right)\right]^{+} \\
& = \begin{cases}0 & \text { se } t \leq S_{1}, \\
\alpha\left(S_{1}\right)\left(t \wedge \tau-S_{1}\right) & \text { se } t>S_{1} .\end{cases}
\end{aligned}
$$


Dessa forma, $A_{\phi}(t)$ é convexa. Por outro lado, no conjunto $\left\{S_{1}>t\right\}$, temos que

$$
\begin{aligned}
\mathbb{P}\left(\left(S_{2}-t\right)^{+}>u \mid \Im_{t}\right) & =\mathbb{P}\left(\left(S_{2}-t\right)^{+}>u \mid S_{1}>t\right) \\
& =\mathbb{P}\left(\left(S_{1}+Y-t\right)^{+}>u \mid S_{1}>t\right) \\
& =\mathbb{P}\left(\left(S_{1}+Y-t\right)^{+}>u \mid\left(S_{1}-t\right)^{+}>0\right) \\
& =\int_{0}^{\infty} \mathbb{P}\left(Y>u-s \mid S_{1}=t+s\right) \exp \{-s-t\} d s \\
& =\int_{0}^{\infty} \exp \left\{-\alpha(t+s)(u-s)^{+}\right\} \exp \{-s-t\} d s
\end{aligned}
$$

Como $\alpha(t)$ é estritamente decrescente em $\left\{S_{1}>t\right\}$, concluímos que $\mathbb{I P}\left((\tau-t)^{+}>\right.$ $u \mid \Im_{t}$ ) é crescente e portanto que $\tau$ não é $I F R \mid \Im_{t}$. 
CAPÍTULO 2

LIMITES DE CONFIABILIDADE PARA SISTEMAS USADOS 


\subsection{Formulação Matemática}

Seja $\tau$ o tempo de vida de um sistema. Note que $\tau$ é um $\Im_{t}$-tempo de parada e que o processo de contagem $N(t)=1_{\{\tau \leq t\}}$ é um $\Im_{t}$-submartingale. Pela decomposição de Doob-Meyer sabemos que existe um único processo $(A(t))_{t \geq 0}, \Im_{t}$-previsível crescente e contínuo a direita, com $A(0)=0$, tal que $N(t)-A(t)$ é um $\Im_{t}$-martingale. $A(t)$ é chamado o $\Im_{t}$-compensador de $N(t)$.

Assuma que para um instante fixado $t$ conhecemos $\left(\Im_{t}\right)_{t \geq 0}$, que pode ser interpretado como o passado dos componentes até o instante $t$ e estamos interessados em estimar a confiabilidade futura do sistema.

Para modelar um sistema usado consideremos um novo espaço de probabilidade $(\Omega, \mathcal{G}, Q) \operatorname{com} \mathcal{G}=\bigvee_{u \geq 0} \mathcal{G}_{u}$, onde

$$
\mathcal{G}_{u}=\sigma\left\{\Im_{t} \bigcup 1_{\{\tau>t+s\}}, \quad 0 \leq s \leq u\right\}
$$

são sub- $\sigma$-álgebras completas e contínuas à direita de $\mathcal{G}=\bigvee_{u \geq 0} \mathcal{G}_{u}$, com $Q\left(A_{1} \mathrm{x} A_{2}\right)=1_{\left\{A_{1}\right\}} \mathbb{P}\left(A_{2} \mid \Im_{t}\right)$ para retângulos $A_{1} \mathrm{x} A_{2} \in \mathcal{G}_{u}$ e $A_{1} \in \Im_{t}$

Podemos interpretar $\mathcal{G}_{u}$ como a história dos componentes até o instante $t$ e o comportamento do sistema no intervalo $(t, t+u]$. Se o processo $N(t)$ conta a falha de um sistema novo, denotaremos $M(u)=\left(N(t+u)-N(t) \mid \Im_{t}\right)$ como o processo que conta a falha de um sistema com idade $t$.

Aplicando novamente a decomposição de Doob-Meyer obteremos o $\mathcal{G}_{u}$-martingale $M(u)-B(u)$, onde $B(u)$ é o $\mathcal{G}_{u}$-compensador de $M(u)$ :

Lema 2.1.1: Sob as condições acima, o $\mathcal{G}_{u}$-compensador de $M(u)$ é dado por

$$
B(u)=\mathbb{E}\left\{A(t+u) \mid \mathcal{G}_{u}\right\}-A(t)
$$


Prova: Note que $\mathcal{G}_{u} \not \subset \mathcal{G}_{0}=\Im_{t}$ e $\mathcal{G}_{u} \subset \Im_{t+u}$ para todo $u \geq 0$. Então para todo $S, \mathcal{G}_{u}$ tempo de parada, temos que

$$
\begin{aligned}
\mathbb{E}_{Q}\{M(S)\} & =\mathbb{E}\left\{N(t+S) \mid \Im_{t}-N(t)\right\} \\
& =\mathbb{E}\left\{A(t+S)-A(t) \mid \Im_{t}\right\} \\
& =\mathbb{E}\left\{\mathbb{E}\left[A(t+S)-A(t) \mid \mathcal{G}_{S}\right] \mid \Im_{t}\right\} \\
& =\mathbb{E}\left\{B(S) \mid \Im_{t}\right\} \\
& =\mathbb{E}_{Q}\{B(S)\}
\end{aligned}
$$

e provamos que $B(u)=\mathbb{E}\left\{A(t+u) \mid \mathcal{G}_{u}\right\}-A(t)$ é o $\mathcal{G}_{u}$-compensador de $M(u)$.

Para prosseguirmos, provaremos que se o $\mathcal{G}_{u}$-compensador $B(u)$ é absolutamente contínuo então podemos escrever

$$
B(u)=-\log \bar{F}\left((t+u \wedge \tau) \mid \Im_{t}\right)
$$

Primeiramente provaremos que

Lema 2.1.2: Se o $\mathcal{G}_{u}$-compensador $B(u)$ de $M(u)$ é absolutamente contínuo $(Q$ q.c.) em $(0, \tau-t]$ então $B(\tau-t)$ é uma variável aleatória com distribuição exponencial de parâmetro 1 .

Prova: Observe que

i) Se $u<\tau-t$, então $M(v)=0,0 \leq v \leq u$, pois o sistema ainda não falhou e, portanto,

$$
\begin{aligned}
1+\int_{0}^{u} i s \exp \{i s B(v)\} d\{B(v)-M(v)\} & =1+\int_{0}^{u} i s \exp \{i s B(v)\} d B(v) \\
& =\exp \{i s B(u)\} \\
& =(1-i s)^{M(u)} \exp \{i s B(u)\}
\end{aligned}
$$


ii) Se $u \geq \tau-t$, então $B(v)=B(\tau-t), \tau-t$ leqv $\leq u$ e $M(\tau-t)=1$, pois o sistema falha no instante $\tau-t$, assim

$$
\begin{aligned}
1+\int_{0}^{u} i s \exp \{i s B(v)\} d\{B(v)-M(v)\}= & 1+\int_{0}^{\tau} i s \exp \{i s B(v)\} d B(v) \\
& -\int_{0}^{u} i s \exp \{i s B(v)\} d M(v) \\
& =1+\exp \{i s B(\tau)\}-1-i s \exp \{i s B(\tau)\} \\
& =(1-i s)^{M(u)} \exp \{i s B(u)\}
\end{aligned}
$$

Portanto concluímos, por $(i)$ e (ii) que

$$
(1-i s)^{M(u)} \exp \{i s B(u)\}=1+\int_{0}^{u} i s \exp \{i s B(v)\} d\{B(v)-M(v)\}
$$

Mas, desde que, $\exp \{i s B(u)\}$ é $\mathcal{G}_{u}$-previsível e $M(u)-B(u)$ é $\mathcal{G}_{u}$-martingale podemos concluir que $(1-i s)^{M(u)} \exp \{i s B(u)\}$ é um martingale com média igual a 1 , e tomando a esperança nos dois lados da equação acima teremos:

$$
\mathbb{E}\{\exp \{i s B(\tau-t)\}\}=\frac{1}{1-i s} .
$$

Teorema 2.1.3: Sob as hipóteses do lema 2.1.2 o $\mathcal{G}_{u}$-compensador de $M(u)$ tem a forma

$$
\left.B(u)=-\log \bar{F}((t+u) \wedge \tau) \mid \Im_{t}\right)
$$

Prova: Seja $U$ um $\mathcal{G}_{u}$-tempo de parada então:

i)se $\tau \leq t+U$,

$$
\mathbb{E}\{B(U)\}=1=\mathbb{E}\left\{-\log \bar{F}\left((t+U) \wedge \tau \mid \Im_{t}\right)\right\}
$$


ii) se $\tau>t+U$, então pelo Teorema T53 Capítulo 3 de Dellacherie (1972) concluímos que $t+U=t+u$ é uma constante $Q$-q.c. e

$$
\begin{aligned}
\mathbb{E}_{Q}\{-\log \bar{F}((t+u) \wedge \tau)\} & =\int_{0}^{\infty} Q\{-\log \bar{F}((t+u) \wedge \tau)>v\} d v \\
& =\int_{0}^{\infty} Q\left\{((t+u) \wedge \tau)>F^{-1}\left(1-\exp ^{-v}\right)\right\} d v \\
& =\int_{0}^{-\log (1-F(t+u))} Q\left\{\tau>F^{-1}\left(1-\exp ^{-v}\right)\right\} d v \\
& =F\left(t+u \mid \Im_{t}\right) .
\end{aligned}
$$




\subsection{Limites para Sistemas com tempo de vida $I F R \mid \Im_{t}$.}

Nesta seção generalizaremos alguns limites para a confiabilidade de sistemas usados com tempo de vida $I F R \mid \Im_{t}$. Desde que o tempo de vida do sistema preserva a propriedade $M I F R \mid \Im_{t}$ de seus componentes podemos assumir esta hipótese e considerar a dependência entre os componentes e sua dinâmica no tempo naturalmente expressa em $\left(\Im_{t}\right)_{t \geq 0}$.

O teorema seguinte nos dá condições em que $\mathcal{G}_{u}$ preserva a propriedade $M I F R \mid \Im_{t}$.

Teorema 2.2.1: Se $\mathbf{S}=\left(S_{1}, \ldots, S_{n}\right)$ é $M I F R \mid \Im_{t}$ e $\theta_{u} \mathbf{S}$ é independente de $\Im_{t+u}$ dado $\mathcal{G}_{u}$, então $\mathbf{S}-t$ é $M I F R \mid \mathcal{G}_{u}$.

Prova: Consideremos um conjunto superior $V$ qualquer, então, para $u \geq u^{*}$ teremos

$$
\begin{aligned}
\mathbb{P}\left(\theta_{u} \mathrm{~S}-t \in V \mid \mathcal{G}_{u}\right) & =\mathbb{P}\left(\theta_{u} \mathrm{~S} \in V+t \mid \mathcal{G}_{u}\right) \\
& =\mathbb{P}\left(\theta_{u} \mathrm{~S} \in V+t \mid \Im_{t+u}\right) \\
& =\mathbb{P}\left(\theta_{t+u} \mathrm{~S} \in V \mid \Im_{t+u}\right) \\
& \leq \mathbb{P}\left(\theta_{t+u^{*}} \mathrm{~S} \in V \mid \Im_{t+u^{*}}\right) \\
& =\mathbb{P}\left(\theta_{u^{*}} \mathrm{~S} \in V+t \mid \mathcal{G}_{u^{*}}\right) \\
& =\mathbb{P}\left(\theta_{u^{*}} \mathrm{~S}-t \in V \mid \mathcal{G}_{u^{*}}\right)
\end{aligned}
$$

e $\mathrm{S}-t$ é $M I F R \mid \mathcal{G}_{u}$.

Quando conhecemos $\mathbb{E}\left(\tau-t \mid \Im_{t}\right)$ para $t$ fixado, um limite inferior para a confiabilidade do sistema é dado pelo seguinte teorema: 
Teorema 2.2.2: Sob as hipótese do Teorema 2.2.1 temos que

$$
\bar{F}\left(t+u \mid \Im_{t}\right) \geq \begin{cases}\exp \left\{\frac{-u}{\boldsymbol{E}\left(\tau-t \mid \Im_{t}\right)}\right\}, & \text { se } u<\mathbb{E}\left(\tau-t \mid \Im_{t}\right) \\ 0, & \text { c.c. }\end{cases}
$$

Prova: Definindo $B^{*}(u)=-\log \bar{F}\left(t+u \mid \Im_{t}\right)$ temos, pelo teorema 1.3.12, que $B^{*}(u)$ é convexa e pelo lema 2.1.2 $B^{*}(\tau-t)=B(\tau-t)$ é uma variável aleatória exponencial com média igual a 1 . Usando a desigualdade de Jensen obtemos que $1=\mathbb{E}_{Q}\left\{B^{*}(\tau-t)\right\} \geq$ $B^{*}\left\{\mathbb{E}_{Q}(\tau-t)\right\}$. Portanto, se $u<\mathbb{E}\left(\tau-t \mid \Im_{t}\right)$ teremos que

$$
\frac{-\log \bar{F}\left(t+u \mid \Im_{t}\right)}{u}=\frac{B^{*}(u)}{u} \leq \frac{B^{*}\left(\mathbb{E}_{Q}(\tau-t)\right)}{\mathbb{E}_{Q}(\tau-t)} \leq \frac{1}{\mathbb{E}_{Q}(\tau-t)}
$$

o que implica que, $\bar{F}\left(t+u \mid \Im_{t}\right) \geq \exp \left\{\frac{-u}{\mathbb{E}\left(\tau-t \mid \Im_{t}\right)}\right\}$.

O teorema anterior pode ser generalizado da seguinte maneira

Teorema 2.2.3 Se $F$ é $I F R \mid \mathcal{G}_{u}$ e $H(v) \geq 0$ é crescente e convexa com $H(u)<$ $\int_{0}^{\infty} H(v) d F\left(v+t \mid \Im_{t}\right)$, então

$$
\int_{0}^{\infty} H(v) d F\left(v+t \mid \Im_{t}\right) \leq \int_{0}^{\infty} H(v) \frac{B^{*}(u)}{u} \exp \left(\frac{-B^{*}(u) v}{u}\right) d v
$$

Prova: Sabemos que $B(u)=-\log \bar{F}\left(t+u \mid \Im_{t}\right)$ é convexa no conjunto $\{t+u<\tau\}$. Assim existe $z, 0 \leq z \leq u$, tal que

$$
\bar{F}\left(t+v \mid \Im_{t}\right) \leq \begin{cases}1, & \text { se } 0 \leq v \leq z \\ \exp \left\{-B^{*}(u) \frac{v-z}{u-z}\right\} & \text { se } v \geq z\end{cases}
$$


Observe que:

$$
\begin{aligned}
\frac{B^{*}(u)}{u-z} \int_{z}^{\infty} H(v) \exp \left\{-B^{*}(u) \frac{v-z}{u-z}\right\} d v= & -\int_{z}^{\infty} H(v)\left\{\exp \left\{-B^{*}(u) \frac{v-z}{u-z}\right\}\right\} d v \\
= & -\left.H(v) \exp \left\{-B^{*}(u) \frac{v-z}{u-z}\right\}\right|_{z} ^{\infty} \\
& +\int_{z}^{\infty} \exp \left\{-B^{*}(u) \frac{v-z}{u-z}\right\} d H(v) \\
= & H(z)+\int_{z}^{\infty} \exp \left\{-B^{*}(u) \frac{v-z}{u-z}\right\} d H(v) \\
\geq & H(z)+\int_{z}^{\infty} \bar{F}\left(t+v \mid \Im_{t}\right) d H(v) \\
= & H(z)+\int_{z}^{\infty} \int_{v}^{\infty} d F\left(t+y \mid \Im_{t}\right) d H(v) \\
= & H(z)+\int_{z}^{\infty} \int_{z}^{y} d H(v) d F\left(t+y \mid \Im_{t}\right) \\
= & H(z)+\int_{z}^{\infty}(H(y)-H(z)) d F\left(t+y \mid \Im_{t}\right) \\
= & H(z)+\int_{z}^{\infty} H(y) d F\left(t+y \mid \Im_{t}\right)-H(z) \bar{F}\left(t+z \mid \Im_{t}\right) \\
= & \int_{z}^{\infty} H(y) d F\left(t+y \mid \Im_{t}\right)+H(z) F\left(t+z \mid \Im_{t}\right)
\end{aligned}
$$

mas

$$
\int_{0}^{z} H(y) d F\left(t+y \mid \Im_{t}\right)=H(z) F\left(t+z \mid \Im_{t}\right)-\int_{0}^{z} F\left(t+y \mid \Im_{t}\right) d H(y) \leq H(z) F\left(t+z \mid \Im_{t}\right)
$$

portanto

$$
\frac{B^{*}(u)}{u-z} \int_{z}^{\infty} H(v) \exp \left\{-B^{*}(u) \frac{v-z}{u-z}\right\} d v \geq \int_{0}^{\infty} H(v) d F\left(t+v \mid \Im_{t}\right)
$$

tomando $\frac{v-z}{u-z}=y \Rightarrow v=z(1-y)+u y$, temos que 


$$
\begin{aligned}
& \int_{0}^{\infty} H(v) d F\left(t+v \mid \Im_{t}\right) \leq B^{*}(u) \int_{0}^{\infty} H(z(1-y)+u y) \exp ^{-y B^{*}(u)} d y \\
& \text { e definindo } \varphi(z)=B^{*}(u) \int_{0}^{\infty} H(z(1-y)+u y) \exp ^{-y B^{*}(u)} d y \text { teremos que } \\
& \alpha \varphi\left(z_{1}\right)+(1-\alpha) \varphi\left(z_{2}\right)= \alpha B^{*}(u) \int_{0}^{\infty} H\left(z_{1}(1-y)+u y\right) \exp ^{-y B^{*}(u)} d y+ \\
&+(1-\alpha) B^{*}(u) \int_{0}^{\infty} H\left(z_{2}(1-y)+u y\right) \exp ^{-y B^{*}(u)} d y \\
& \geq B^{*}(u) \int_{0}^{\infty} H\left(\alpha z_{1}(1-y)+\alpha u y+\right. \\
&\left.+(1-\alpha) z_{2}(1-y)+(1-\alpha) u y\right) \exp ^{-y B^{*}(u)} d y \\
&= B^{*}(u) \int_{0}^{\infty} H\left(\left(\alpha z_{1}+(1-\alpha) z_{2}\right)(1-y)+u y\right) \exp ^{-y B^{*}(u)} d y \\
&= \varphi\left(\alpha z_{1}+(1-\alpha) z_{2}\right)
\end{aligned}
$$

e concluímos que $\varphi(z)$ é convexa.

Suponha ainda que $\varphi(z)>\varphi(0)$ e $\varphi(z)>\varphi(u)$, para $\alpha$ tal que $(1-\alpha) u=z$, então:

$$
\begin{aligned}
\alpha \varphi(0)+(1-\alpha) \varphi(u) & \leq \alpha \varphi(z)+(1-\alpha) \varphi(z) \\
& =\varphi(z)=\varphi(0 . \alpha+(1-\alpha) \cdot u)
\end{aligned}
$$

que é absurdo pois $\varphi$ é convexa, mas

$$
\varphi(u)=B^{*}(u) \int_{0}^{\infty} H(u) \exp ^{-y B^{*}(u)} d y=H(u) \geq \varphi(z) \geq \int_{0}^{\infty} H(v) d F\left(t+v \mid \Im_{t}\right)
$$

é um outro absurdo, por hipótese. Logo

$$
\begin{aligned}
\varphi(0)=\int_{0}^{\infty} B^{*}(u) H(u y) \exp ^{-y B^{*}(u)} d y & =\int_{0}^{\infty} H(v) \frac{B^{*}(u)}{u} \exp \frac{-B^{*}(u) v}{u} d v \\
& \geq \varphi(z) \geq \int_{0}^{\infty} H(v) d F\left(t+v \mid \Im_{t}\right)
\end{aligned}
$$


Uma aplicação imediata do teorema acima é dada por

Corolário 2.2.4 Se $F$ é $I F R \mid \mathcal{G}_{u}$ e $r \geq 1$ com $\mu_{r}=\int_{0}^{\infty} v^{r} d F\left(v+t \mid \Im_{t}\right)$, então

$$
\bar{F}\left(t+u \mid \Im_{t}\right) \geq \exp \left(\frac{-\mu}{\lambda_{r}^{\frac{1}{r}}}\right) \text { se } u<\left[\lambda_{r} \Gamma(r+1)\right]^{\frac{1}{r}}
$$

onde $\lambda_{r}=\frac{\mathbb{E}\left[(\tau-t)^{r} \mid \Im_{t}\right]}{\Gamma(r+1)}$.

Prova: Note que $H(v)=v^{r}$ é crescente e convexa e que $u^{r}<\int_{0}^{\infty} v^{r} d F(v+$ $\left.t \mid \Im_{t}\right) \Leftrightarrow u<\left[\lambda_{r} \Gamma(r+1)\right]^{\frac{1}{r}}$ então, pelo teorema 2.2.3, temos que

$$
\begin{gathered}
\int_{0}^{\infty} v^{r} d F\left(v+t \mid \Im_{t}\right) \leq \int_{0}^{\infty} v^{r} \frac{B^{*}(u)}{u} \exp \left(\frac{-B^{*}(u) v}{u}\right) d v \Rightarrow \\
\mu_{r} \leq \frac{\Gamma(r+1)}{\frac{B^{*}(u)^{r}}{u}} \int_{0}^{\infty} \frac{v^{r}\left(\frac{B^{*}(u)}{u}\right)^{r+1} \exp \left(\frac{-B^{*}(u) v}{u}\right) d v}{\Gamma(r+1)} \Rightarrow \\
\mu_{r} \leq \frac{\Gamma(r+1)}{\left(\frac{B^{*}(u)}{u}\right)^{r}} \Rightarrow \frac{B^{*}(u)}{u} \leq \frac{1}{\lambda_{r}^{\frac{1}{r}}} \Rightarrow \\
-\log \bar{F}\left(t+u \mid \Im_{t}\right) \leq \frac{u}{\lambda_{r}^{\frac{1}{r}}} \Rightarrow \bar{F}\left(t+u \mid \Im_{t}\right) \geq \exp \left(\frac{-u}{\lambda_{r}^{\frac{1}{r}}}\right)
\end{gathered}
$$

Se o sistema consiste de $n$ componentes em série e conhecemos $\mathbb{E}\left(S_{i}-t \mid \Im_{t}\right)$ para cada $i, 1 \leq i \leq n$, podemos obter o limite clássico: 
Teorema 2.2.5: Considere um sistema de $n$ componentes em série com tempos de vida $S_{1}, \ldots, S_{n}$ tal que $\left(S_{1}, \ldots, S_{n}\right)$ seja $M I F R \mid \Im_{t}$ e $\mathbb{P}\left\{S_{i} \neq S_{j}\right\}=1$ com $1 \leq i \leq n$, $1 \leq j \leq n$ e $i \neq j$. Então

$$
\bar{F}\left(t+u \mid \Im_{t}\right) \geq \begin{cases}\exp \left\{-u \sum_{i=1}^{n} \frac{1}{\mathbb{E}\left(S_{i}-t \mid \Im_{t}\right)}\right\}, & \text { se } u<m \\ 0, & \text { c.c. }\end{cases}
$$

onde $m=\min _{\{1 \leq i \leq n\}}\left\{\mathbb{E}\left(S_{i}-t \mid \Im_{t}\right)\right\}$

Prova: Primeiramente, observe que para um sistema formado por componentes em série temos que $\Im_{t+u}=\mathcal{G}_{u}$ para todo $u$ e as condições do Teorema 2.2 .1 estão satisfeitas.

Baseado no Teorema 4.1 de Arjas (1981 a) podemos concluir que cada $S_{i}, i=$ $1, \ldots, n$ é $I F R \mid \mathcal{G}_{u}$ e portanto cada $\mathrm{B}_{i}(u)$ (o $\mathcal{G}_{u}$-compensador do submartingale $M_{i}(u)=$ $\left.N_{i}\left(t+u \mid \Im_{t}\right)-N_{i}(t)\right)$ é convexo $(Q-$ q.c. $)$ em $\left(0, S_{i}-t\right]$, e portanto, cada $B_{i}^{*}(u)$ será convexa.

Pelo Lema 1.3.4 de Arizono (1992) sabemos que os $\mathcal{G}_{u}$-compensadores $B_{i}\left(S_{i}-\right.$ $t)], i=1, \ldots, n$ são variáveis aleatórias exponenciais independentes e identicamente distribuídas com média igual a 1 (mesmo que os tempos de vida $S_{i}, i=1, \ldots, n$ não sejam independentes).

Usando a desigualdade de Jensen temos que

$$
1=\mathbb{E}_{Q}\left\{B_{i}^{*}\left(S_{i}-t\right)\right\} \geq B_{i}^{*}\left\{\mathbb{E}\left(S_{i}-t \mid \Im_{t}\right)\right\}
$$


Mas, o $\mathcal{G}_{u}$-compensador de um sistema em série pode ser decomposto como $\left.B_{(}^{*} u\right)=$ $\sum_{i=1}^{n} B_{i}^{*}(u)$ e portanto

$$
\begin{aligned}
\frac{B^{*}(u)}{u} & =\frac{\sum_{i=1}^{n} B_{i}^{*}(u)}{u} \\
& \leq \frac{\sum_{i=1}^{n} B_{i}^{*}\left(\mathbb{E}\left(S_{i}-t \mid \Im_{t}\right)\right)}{\mathbb{E}\left(S_{i}-t \mid \Im_{t}\right)} \\
& \leq \sum_{i=1}^{n} \frac{1}{\mathbb{E}\left(S_{i}-t \mid \Im_{t}\right)}
\end{aligned}
$$

$\log 0$

$$
\begin{gathered}
\frac{-\log \bar{F}\left(t+u \mid \Im_{t}\right)}{u} \leq \sum_{i=1}^{n} \frac{1}{\mathbb{E}\left(S_{i}-t \mid \Im_{t}\right)} \quad \text { e } \\
\bar{F}\left(t+u \mid \Im_{t}\right) \geq \exp \left\{-\sum_{i=1}^{n} \frac{u}{\mathbb{E}\left(S_{i}-t \mid \Im\right)}\right\}, \quad \text { se } \quad u<m
\end{gathered}
$$

Em um experimento amostral os tempos de vida de alguns itens podem ser censurados e, portanto, uma estimativa da média não pode ser obtida, contudo sempre será possível estimar alguns percentis. Podemos então obter limites para a confiabilidade do sistema como se segue. 
O resultado seguinte é similar ao resultado obtido por Barlow e Proschan (1981) para limites com percentil conhecido.

Teorema 2.2.6: Sob as condições do Teorema 2.2.1, se $\mathbb{P}\left(\tau \leq t+\xi_{p} \mid \Im_{t}\right)=p$, então

$$
\bar{F}\left(t+u \mid \Im_{t}\right) \begin{cases}\geq \exp \{-\alpha u\}, & \text { se } u \leq \xi_{p} \\ \leq \exp \{-\alpha u\}, & \text { se } u>\xi_{p}\end{cases}
$$

onde $\alpha=\frac{-\log (1-p)}{\xi_{p}}$.

Prova: Desde que $B^{*}(u)$ é $Q$-q.c. convexo, se $0<u \leq \xi_{p}$ então

$$
\bar{F}^{\frac{1}{u}}\left(t+u \mid \Im_{t}\right) \geq \bar{F}^{\frac{1}{\xi_{p}}}\left(t+\xi_{p} \mid \Im_{t}\right)
$$

e, portanto,

$$
\bar{F}\left(t+u \mid \Im_{t}\right) \geq \bar{F}^{\frac{u}{\xi_{p}}}\left(t+\xi_{p} \mid \Im_{t}\right)=(1-p)^{\frac{u}{\xi_{p}}}=\exp \{-\alpha u\}
$$

Se $u \geq \xi_{p}$ a prova segue de maneira análoga.

Os resultados seguintes fornecem limites para os momentos de uma distribuição FIF $R \mid \mathcal{G}_{u}$.

Teorema 2.2.7: Se $F$ é $I F R \mid \mathcal{G}_{u}$, com média $m_{t}=\mathbb{E}\left(\tau-t \mid \Im_{t}\right)$ e se $\varphi(v)$ é crescente (decrescente), então

$$
\int \varphi(v) \bar{F}\left(t+v \mid \Im_{t}\right) d v \leq(\geq) \int \varphi(v) \exp \left\{\frac{-v}{m_{t}}\right\} d v
$$

Prova: Se $F\left(t+v \mid \Im_{t}\right)$ é identicamente distribuída à exponencial $\frac{\exp \left(\frac{-v}{m_{t}}\right)}{m_{t}}$ então vale a igualdade. 
Caso contrário como $-\log \bar{F}\left(t+v \mid \Im_{t}\right)$ é convexa, $F\left(t+v \mid \Im_{t}\right)$ com média $m_{t}$, $\bar{F}\left(t+v \mid \Im_{t}\right)$ cruza $\exp \left(\frac{-v}{m_{t}}\right)$ em algum ponto, digamos $v_{0}$, então

$$
\begin{aligned}
& \int \varphi(v) \bar{F}\left(t+v \mid \Im_{t}\right) d v-\int \varphi(v) \exp \left(\frac{-v}{m_{t}}\right) d v=\int\left(\varphi(v)-\varphi\left(v_{0}\right)\right)\left(\bar{F}\left(t+v \mid \Im_{t}\right)-\exp \left(\frac{-v}{m_{t}}\right)\right) d v \\
& \leq(\geq) 0
\end{aligned}
$$

Corolário 2.2.8: Se $F$ é $I F R \mid \mathcal{G}_{u}$ e $\mu_{r}=\int u^{r} d F\left(t+u \mid \Im_{t}\right)$, então

$$
\mu_{r}\left\{\begin{array}{l}
\leq \Gamma(r+1) m_{t}^{r}, \text { se } r \geq 1 \\
\geq \Gamma(r+1) m_{t}^{r}, \text { se } 0 \leq r \leq 1
\end{array}\right.
$$

e

$$
\int \exp ^{-s v} d F\left(t+v \mid \Im_{t}\right) \leq \frac{1}{1+s m_{t}}
$$

Prova: Seja $\varphi(v)=v^{r-1}$. Temos que $\varphi^{\prime}(v)=(r-1) v^{r-2}$ de maneira que se $r \geq 1, \varphi(v)$ é crescente. Contudo

$$
\begin{aligned}
\mu_{r} & =\int_{0}^{\infty} u^{r} d F\left(t+u \mid \Im_{t}\right) \\
& =\int_{0}^{\infty}\left(\int_{0}^{u} r v^{r-1} d v\right) d F\left(t+u \mid \Im_{t}\right) \\
& =\int_{0}^{\infty} r v^{r-1} \int_{v}^{\infty} d F\left(t+u \mid \Im_{t}\right) d v \\
& =r \int_{0}^{\infty} v^{r-1} \bar{F}\left(t+v \mid \Im_{t}\right) d v
\end{aligned}
$$

Aplicando o teorema anterior, temos que

$$
\begin{aligned}
\mu_{r} & =r \int_{0}^{\infty} v^{r-1} \bar{F}\left(t+v \mid \Im_{t}\right) d v \\
& \leq r \int_{0}^{\infty} v^{r-1} \exp \left(\frac{-v}{m_{t}}\right) d v \\
& =\Gamma(r+1) m_{t}^{r} .
\end{aligned}
$$


Se $0 \leq r \leq 1, \varphi(v)$ é decrescente e a prova é semelhante.

Note que

$$
\begin{aligned}
\int_{0}^{\infty} \exp (-s v) \bar{F}\left(t+v \mid \Im_{t}\right) d v & =\int_{0}^{\infty} \int_{0}^{u} \exp (-s v) d v d F\left(u+t \mid \Im_{t}\right) \\
& =\int_{0}^{\infty} \frac{1-\exp (-s u)}{s} d F\left(t+u \mid \Im_{t}\right)
\end{aligned}
$$

Como $\exp (-s u)$ é decrescente em $u$, para $s>0$, utilizamos o teorema anterior e concluímos que

$$
\int_{0}^{\infty} \frac{1-\exp (-s u)}{s} d F\left(t+u \mid \Im_{t}\right) \geq \int_{0}^{\infty} \exp (-s u) \exp \left(\frac{-u}{m_{t}}\right) d u=\frac{1-\frac{1}{1+s m_{t}}}{s}
$$

e, portanto

$$
\int_{0}^{\infty} \exp (-s u) d F\left(t+u \mid \Im_{t}\right) \leq \frac{1}{1+s m_{t}}
$$


2.3 Limites para Sistemas com Tempo de vida $N B U \mid \Im_{t}$.

Neste trabalho preservaremos a interpretação intuitiva do compensador como uma função do risco condicional. Como a propriedade $N B U \mid \mathcal{G}_{u}$ não é suficiente para a continuidade da função risco, o compensador não terá a forma $B(u)=-\log \bar{F}\left((t+u) \wedge \tau \mid \Im_{t}\right)$, e não poderemos usar esta propriedade.

Observe que se $\left(S_{1}, \ldots, S_{n}\right)$ é $\quad M I F R \mid \Im_{t}$ então $\tau$ é $I F R \mid \Im_{t}$ e consequentemente é $N B U \mid \Im_{t}$. Podemos provar também que

Teorema 2.3.1: Se $\left(S_{1}, \ldots, S_{n}\right)$ é $M I F R \mid \Im_{t}$, então o tempo de vida do sistema usado $\left(\tau-t \mid \Im_{t}\right)$ é $N B U \mid \mathcal{G}_{u}$.

Prova: Note que

$$
\begin{aligned}
Q\left(\tau-u-t>s \mid \mathcal{G}_{u}\right) & =\mathbb{E}\left(\mathbb{E}\left(1_{\{\tau-t-u>s\}} \mid \Im_{t}\right) \mid \mathcal{G}_{u}\right) \\
& =\mathbb{E}\left(\mathbb{E}\left(\mathbb{E}\left(1_{\{\tau-t-u>s\}} \mid \Im_{t+u}\right) \mid \Im_{t}\right) \mid \mathcal{G}_{u}\right) \\
& \leq \mathbb{E}\left(\mathbb{E}\left(\mathbb{E}\left(1_{\{\tau-t>s\}} \mid \Im_{t}\right) \mid \Im_{t}\right) \mid \mathcal{G}_{u}\right) \\
& =\mathbb{E}\left(\mathbb{E}\left(1_{\{\tau-t>s\}} \mid \Im_{t}\right) \mid \mathcal{G}_{u}\right) \\
& =\mathbb{E}\left(\mathbb{E}\left(1_{\{\tau-t>s\}} \mid \mathcal{G}_{0}\right) \mid \mathcal{G}_{u}\right) \\
& =\mathbb{E}\left(1_{\{\tau-t>s\}} \mid \mathcal{G}_{0}\right) \\
& =Q\left(\tau-t>s \mid \mathcal{G}_{0}\right)
\end{aligned}
$$

Para obtermos limites para a confiabilidade de sistemas com tempos de vida $N B U \mid \mathcal{G}_{u}$, consideraremos a classe

$$
\Sigma=\left\{F: F \quad \text { é } \quad N B U \mid \mathcal{G}_{u} \quad \text { e } \quad \int_{0}^{\infty} \bar{F}\left(t+s \mid \Im_{t}\right) d s=m_{t}\right\}
$$

onde $m_{t}=\mathbb{E}\left(\tau-t \mid \Im_{t}\right)$. 
Teorema 2.3.2: Se $\left(S_{1}, \ldots, S_{n}\right)$ é $M I F R \mid \Im_{t}$ e se $\int_{0}^{\infty} \bar{F}\left(t+s \mid \Im_{t}\right) d s=m_{t}$ então

$$
\inf _{F \in \Sigma} \bar{F}\left(t+u \mid \Im_{t}\right)= \begin{cases}1-\frac{u}{m_{t}}, & \text { se } u<m_{t} \\ 0, & \text { se } u \geq m_{t}\end{cases}
$$

Prova: Escolha um elemento $F$ de $\Sigma$. Para $u<m_{t}$ fixo, definiremos a nova função de sobrevivência

$$
\bar{G}_{\alpha}\left(t+v \mid \Im_{t}\right)=(\alpha)^{n} \text { se } n u \leq v<(n+1) u \text { para } n=0,1,2, \ldots
$$

Podemos provar que $G_{\alpha}$ é $N B U \mid G_{u}$, e que sua esperança é igual a $h(\alpha)=\frac{u}{1-\alpha}$.

Então, para $n u \leq v<(n+1) u$ e $\alpha=\bar{F}\left(t+u \mid \Im_{t}\right)$, teremos

$$
\bar{F}\left(t+v \mid \Im_{t}\right) \leq \bar{F}\left(t+n u \mid \Im_{t}\right) \leq\left(\bar{F}\left(t+u \mid \Im_{t}\right)\right)^{n}=\bar{G}_{\alpha}\left(v+t \mid \Im_{t}\right)
$$

e, portanto

$$
\begin{aligned}
u<m_{t} & =\int_{0}^{\infty} \bar{F}\left(t+v \mid \Im_{t}\right) d v \\
& =\sum_{n=0}^{\infty} \int_{n u}^{(n+1) u} \bar{F}\left(t+v \mid \Im_{t}\right) d v \\
& \leq \sum_{n=0}^{\infty} \int_{n u}^{(n+1) u}\left(\bar{F}\left(t+v \mid \Im_{t}\right)\right)^{n} d v \\
& =\frac{u}{1-\bar{F}\left(t+u \mid \Im_{t}\right)} .
\end{aligned}
$$

Então, se $\alpha$ decresce continuamente para $0, \frac{u}{1-\alpha}$ decresce continuamente para $u$ e, portanto, existe um $\alpha^{*}$ tal que $m_{t}=\frac{u}{1-\alpha^{*}}$, ou seja,

$$
\alpha^{*}=\frac{m_{t}-u}{m_{t}}=1-\frac{u}{m_{t}}
$$


mas, observe que a função

$$
\bar{G}_{\alpha^{*}}\left(t+v \mid \Im_{t}\right)=\left(\frac{u-m_{t}}{m_{t}}\right)^{n} \text { se } n u \leq v<(n+1) u \text { para } n=0,1,2, \ldots
$$

pertence a $\Sigma$, desde que

$$
h\left(\alpha^{*}\right)=m_{t} \leq \frac{u}{1-\bar{F}\left(t+u \mid \Im_{t}\right)}=h\left(\bar{F}\left(t+u \mid \Im_{t}\right)\right)
$$

chegamos na desigualdade $\alpha^{*} \leq \bar{F}\left(t+u \mid \Im_{t}\right)$. Como a escolha da $F$ foi arbitrária, o teorema está provado. 


\section{BIBLIOGRAFIA}

[01] Arizono, H. (1992) . Martingais e Teoria da Confiablidade. Dissertação apresentada ao Instituto de Matemática e Estatística - USP.

[02] Arizono, H \& Bueno V.C. (1997) . Bounds on system reliability of used components under $M I F R \mid \Im_{t}$ assumption.Statistics \& Probability Letters, 36: 49-57.

[03] Arjas, E. (1981a) . A stochastic process approach to multivariate reliability systems: notions based on conditional stochastic order. Mathematics of Operations Research, 6(2): 263-276.

[04] Arjas, E. (1981b) . The failure and hazard process in multivariate reliability systems. Mathematics of Operations Research, 6(4) : 551-562.

[05] Barlow, R. \& Proschan, F. (1981). Statistical Theory of Reliability and Life Testing. To Begin With. Silver Spring, MD.

[06] Bremaud (1980). Point Processes and Queues: martingales dynamics. SpringerVerlag, New York.

[07] Brown, T. (1978) . A martingale approach to the Poisson convergence of simple point processes. The Annals of Probability, 6(4) : 615-628. 
[08] Jacod, J. (1975) . Multivariate point processes: predictable projection, RadonNikodym derivatives, representation of martingales. $Z$ Wahrscheinlichkeitstheorie Verw. Gebiete,31 : 235-253.

[09] Norros, I. (1986) . A compensator representation of multivariate life lenght distributions, with applications. Scandinavian Journal of statistics, 13 : 99-112.

[10] Liptser, R.S. \& Shiryayev A.N. (1977a). Statistics of Random Processes I General Theory, Springer - Verlag, NY.

[11] Liptser, R.S. \& Shiryayev A.N. (1977b) . Statistics of Random Processes II Applications, Springer - Verlag, NY.

[12] Dellacherie (1972) . Capacités et Processus Stochastiques, Springer, NY.

[13] Breiman L. (1968) . Probability, Addison-Wesley Publishing Company. 